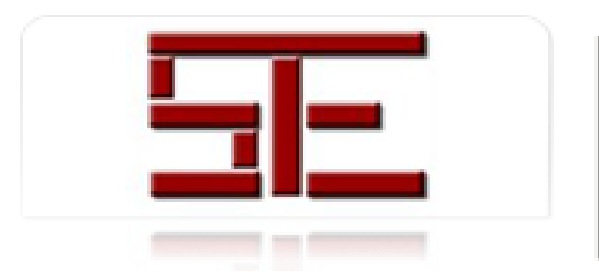

Teoría de la Educación. Educación y Cultura en la Sociedad de la Información

E-ISSN: 1138-9737

revistatesi@usal.es

Universidad de Salamanca

\title{
España
}

McAnally-Salas, Lewis; Lavigne, Gilles; Organista Sandoval, Javier MARCO DE REFERENCIA PARA DISEÑAR CURSOS EN ADMINISTRACIÓN: EL CASO DE CULTURA ORGANIZACIONAL

Teoría de la Educación. Educación y Cultura en la Sociedad de la Información, vol. 14, núm. 2, 2013, pp. 228-251

Universidad de Salamanca

Salamanca, España

Disponible en: http://www.redalyc.org/articulo.oa?id=201028055010

Cómo citar el artículo

Número completo

- Más información del artículo

Página de la revista en redalyc.org

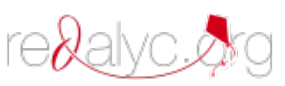

Sistema de Información Científica

Red de Revistas Científicas de América Latina, el Caribe, España y Portugal Proyecto académico sin fines de lucro, desarrollado bajo la iniciativa de acceso abierto 


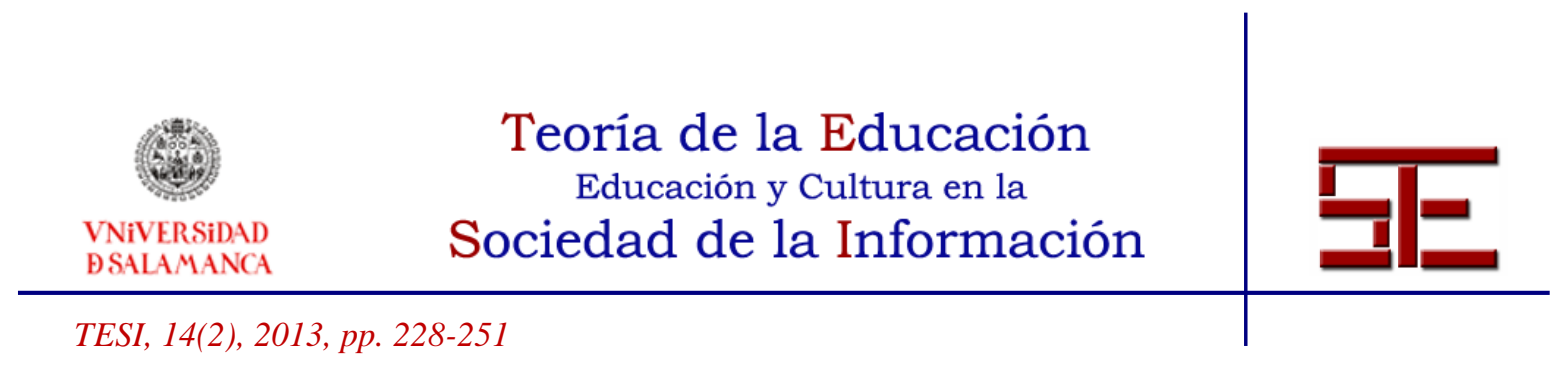

\section{MARCO DE REFERENCIA PARA DISEÑAR CURSOS EN ADMINISTRACIÓN: EL CASO DE CULTURA ORGANIZACIONAL}

Resumen: Se presenta un marco de trabajo para diseñar cursos, se aborda específicamente el caso de cursos de cultura organizacional. Se hace una pequeña descripción de las principales teorías del aprendizaje y se describe a mayor detalle las características de ambientes de aprendizaje constructivistas. Se propone la utilización de un modelo almenado como plantilla para programar actividades y contenidos, el cual, cuando se utiliza conjuntamente con tecnologías educativas para transmitir el contenido explícito del curso, permite optimizar el tiempo de clases cara a cara permitiendo más tiempo para la transferencia de conocimiento tácito. Se discuten seis consideraciones para el diseño de cursos de cultura organizacional tomando como base las propuestas de Lundberg y se hacen recomendaciones para darles una orientación constructivista a los cursos.

Palabras clave: diseño educativo; cultura organizacional; tecnología educativa.

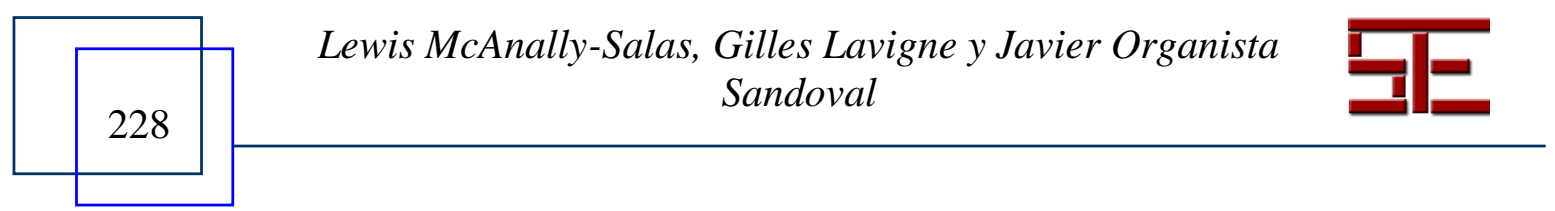




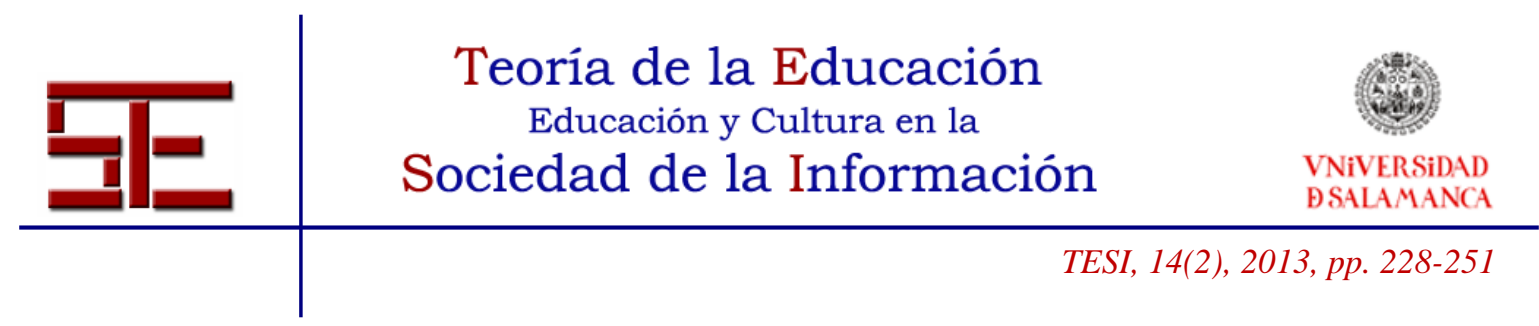

\title{
RAMEWORK FOR DESIGNING ADMINISTRATION COURSES: THE ORGANIZATIONAL CULTURE CASE
}

\begin{abstract}
A framework to courses design is propose, specifically addresses the organizational culture courses. A short description of the main learning theories is made and describes in greater detail the characteristics of constructivist learning environments. A castle-top model as a template for programming activities and contents is proposed, which, when used in conjunction with educational technologies to take care of explicit knowledge, the face to face activities are optimize, allowing more time for the transfer of tacit knowledge. Base in the proposal made by Lundberg, six considerations for the design of organizational culture courses are discussed and recommendations are made to give a constructivist orientation courses.
\end{abstract}

Keywords: instructional design; organizational culture; educational technology.

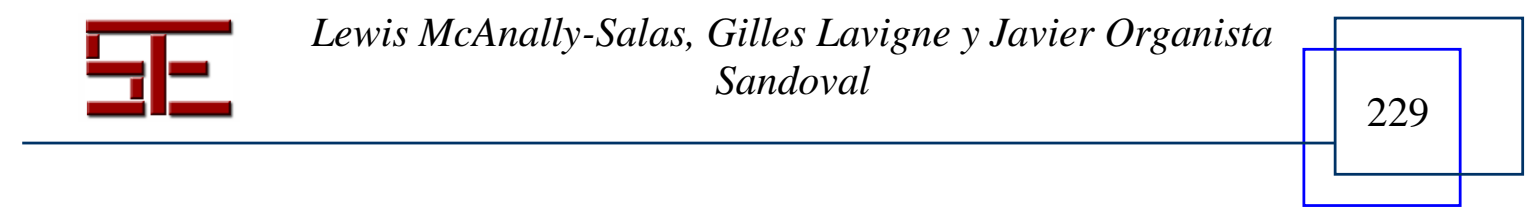




\begin{tabular}{c|c} 
Teoría de la Educación \\
$\begin{array}{c}\text { Educación y Cultura en la } \\
\text { VNiVERSIDAD } \\
\text { BSALAMANCA }\end{array}$ \\
\hline TESI, 14(2), 2013, pp. 228-251
\end{tabular}

\section{MARCO DE REFERENCIA PARA DISEÑAR CURSOS EN ADMINISTRACIÓN: EL CASO DE CULTURA ORGANIZACIONAL}

Fecha de recepción: 04/03/2012; fecha de aceptación: 01/03/2013; fecha de publicación: 30/07/2013

Lewis McAnally-Salas

mcanally@uabc.edu.mx

Universidad Autónoma de Baja California

Gilles Lavigne

gilles@uabc.edu.mx

Universidad Autónoma de Baja California

Javier Organista Sandoval

javor@uabc.mx

Universidad Autónoma de Baja California

\section{1.- INTRODUCCIÓN}

Las demandas que la globalización impone sobre los profesionistas es a su vez una demanda que el contexto ejerce sobre las Instituciones de Educación Superior (IES). En un mundo fuertemente globalizado donde las fronteras de la competencia ya no corresponden a las fronteras geográficas, es necesario revisar los fundamentos tradicionales y verificar su alineación con las demandas de formación e información a los profesionistas en los albores del siglo XXI. La educación superior sufre fuertes presiones sociales y económicas para su transformación (Katz \& Associates, 1999; Katz, EDUCAUSE (Association), \& PricewaterhouseCoopers, 1999; Oblinger \& Rush, 1997), en la nueva era del conocimiento se identifica un nuevo modelo para la producción del conocimiento (modo 2) que responde preferentemente a las demandas sociales, industriales y empresariales a diferencia de responder a una visión más academicista (modo 1) (ver Gibbons, 1994). Simultáneamente con el surgimiento de diferentes modelos de generar conocimiento, el uso de las tecnologías de la información y comunicación (TIC) se fue imponiendo como uno de las principales componentes de la sociedad de conocimiento, de hecho, la ubicuidad de las TIC, principalmente Internet, es lo que ha permitido el surgimiento de nuevos modelos de gestión del conocimiento y de

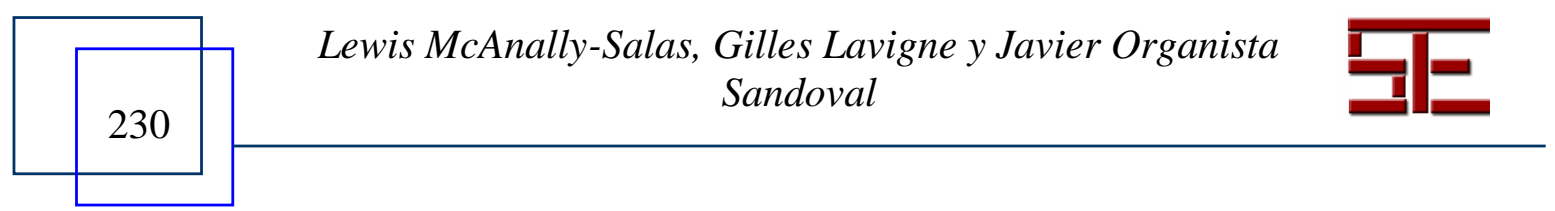




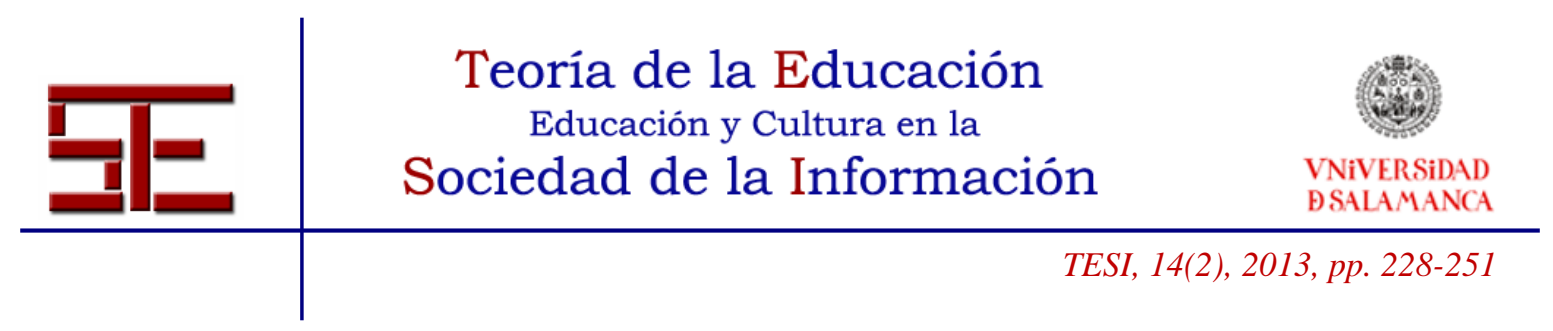

la nueva economía basada en el conocimiento (Clarke \& Clegg, 1998; Despres \& Chauvel, 2000; Lam, 2000; Nonaka \& Takeuchi, 1995; Castells, 2008).

En particular los programas de Administración se han transformado profundamente. Han cambiado fundamentalmente desde la primera mitad del siglo pasado en que las escuelas carecían de profesionalización, a sufrir una fuerte profesionalización en la década de los 60 , y en los 90 se han enfrentado a la competencia global. Actualmente la búsqueda es adecuarse a la formación de profesionistas con un pensamiento integrador, opuesto a la excesiva especialización que no le permite al profesionista ser capaz de tener una visión completa del proceso en cuestión, sea contable, de mercadotecnia o financiero (Latham, Latham, \& Whyte, 2004). Es necesario reconocer que la incertidumbre y la ambigüedad es inherente a la administración, y se debe asumir como tal, evitando visiones sobresimplificadas de la misma, incorporando la complejidad como elemento fundamental en la formación profesional (Cunha, Cunha, \& Cabral-Cardoso, 2004). A decir de Robert B. Reich (1992a), se necesita la formación de analistas simbólicos en una nueva economía en donde el conocimiento, no los datos o la información, es la moneda de curso legal. Las principales habilidades de este nuevo profesionista es la conceptualización de problemas y soluciones mediante el desarrollo de cuatro competencias básicas; (a) capacidad de abstracción en un mundo complejo, (b) capacidad de pensamiento sistémico en un mundo analítico, (c) capacidad de experimentación y (d) capacidad de colaborar en equipo, en un mundo individualizado (Reich, 1992b).

Para lograr lo planteado, es necesario tener una aproximación mucho más holista al diseño educativo de los cursos con una fuerte integración de la computadora en el proceso. Bajo estas demandas, ya no es posible pretender diseñar cursos químicamente puros, bajo una sola teoría del aprendizaje.

La comprensión de los principios básicos en que se sustentan las principales teorías del aprendizaje nos permite su aplicación de manera coherente y complementaria.

\section{1.- Principales teorías del aprendizaje}

La escuela de pensamiento conductista, influenciada por Thorndike, Pavlov y Skinner, postula que el aprendizaje es un cambio en la conducta que puede ser observable, y que es causada por estímulos externos del ambiente. Así pues, es la conducta observable la que indica si el aprendiz ha aprendido algo, por lo que el interés se centra en las acciones del sujeto, no lo que ocurre en su cabeza (Ally, 2004; Roblyer \& Edwards, 2000). Lo

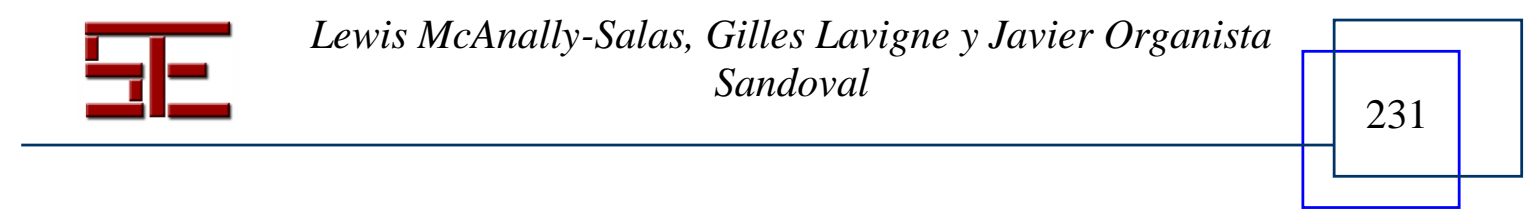




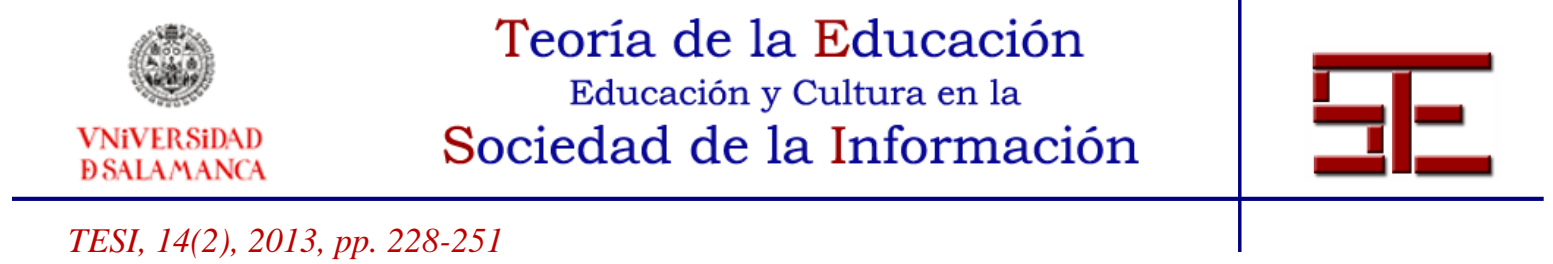

anterior ha tenido implicaciones en la forma como es percibido el conocimiento, es decir, el conocimiento está dado y es conocido, por lo tanto es posible definir lo que es y predecir sus resultados cuando es aprendido. Esta aproximación está relacionada con estrategias centradas en el profesor y la enseñanza, en donde el profesor es el que sabe y el que dice qué hay que saber, utilizando los métodos más apropiados para lograr los objetivos de aprendizaje.

Como es de esperar, algunos investigadores aseguraban que el aprendizaje va más allá de conductas observables y buscaron alternativas en las teorías de aprendizaje cognitivas. La psicología cognitiva plantea que el aprendizaje involucra la memoria, la motivación y el pensamiento, y que la reflexión juega un papel importante en el aprendizaje. Los seguidores de estas teorías conciben el aprendizaje como un proceso interno, y que la capacidad de aprendizaje depende de factores como la cantidad de esfuerzo invertido, capacidad y profundiad de procesamiento, y las estructuras de conocimiento existentes (Ally, 2004; Roblyer \& Edwards, 2000).

La tendencia más reciente es el constructivismo, cuyos teóricos plantean que el aprendiz interpreta la información y el mundo a su alrededor de acuerdo a su realidad personal, aprendiendo por observación, procesamiento e interpretación, pasando finalmente a personalizar la información como conocimiento personal. Bajo esta aproximación se asegura que el sujeto aprende cuando puede contextualizar para una aplicación inmediata y para darle significado. El sujeto construye el conocimiento y el profesor tiene un papel de facilitador, es decir, es el responsable de guiar al estudiante y brindarle múltiples recursos para la apropiación del conocimiento.

Ally (2004) menciona que cuando estas tres aproximaciones se observan con detenimiento, en muchos puntos existen traslapes en sus ideas y principios. Lo anterior tiene implicaciones fundamentales en el diseño educativo de los cursos, ya que permite integrar la aproximación más idónea para el tipo de contenido y meta de aprendizaje del curso. De manera general, el conductismo presenta estrategias apropiadas cuando en una unidad o tema la pregunta primordial es ¿qué?, típica de cuando se busca que se aprendan hechos. Cuando la pregunta primordial es ¿cómo?, para procesos y principios la mejor aproximación es el cognitivismo, y cuando la pregunta es del tipo ¿por qué?, que orienta a procesamiento de alto nivel, la aproximación adecuada es el constructivismo.

Con base en lo anterior no debemos concluir que el diseño de los cursos no debe privilegiar una teoría en particular, lo que debe quedar claro es que, privilegiando una de

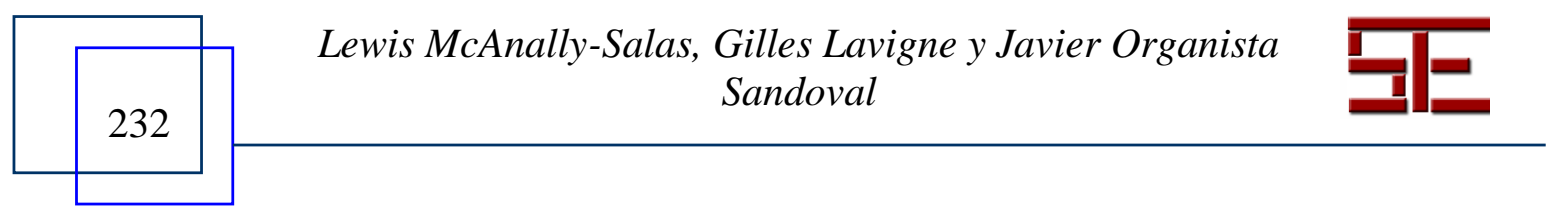




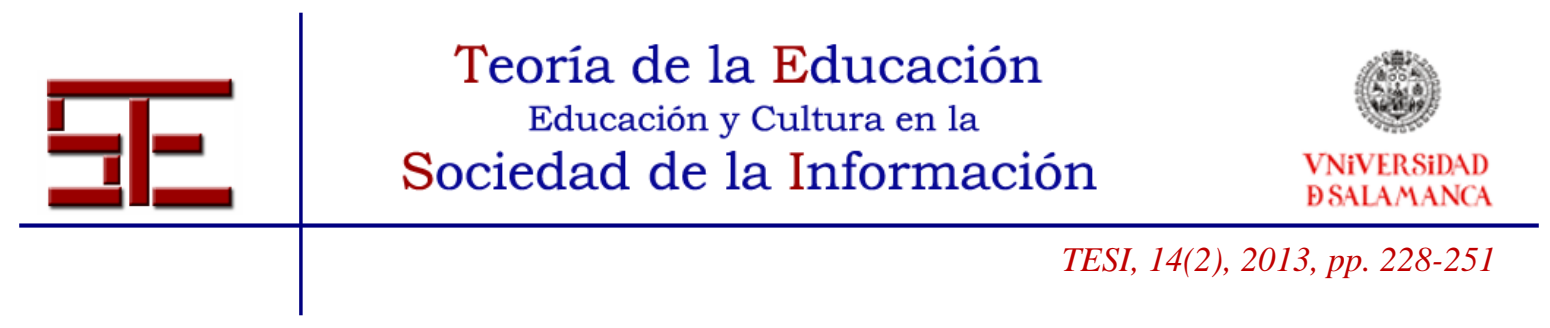

ellas, no se deben excluir las demás buscando la pureza del diseño. Las metas de aprendizaje deben ser las guías indicadoras para privilegiar una u otra teoría, volviendo a recalcar que es conveniente adherirse a una de ellas como la teoría principal, sobre la que se hace el diseño general.

Por las tendencias y necesidades mencionadas con anterioridad para las carreras de Administración, en este trabajo se plantea que el constructivismo es la teoría del aprendizaje cuyas estrategias permiten el desarrollo de habilidades y competencias que se demandan en estos principios del siglo XXI.

\section{2.- Estrategias constructivistas}

Considerando las habilidades del analista simbólico que presenta Reich (1992a) para este nuevo profesionista, el cual requiere desarrollar cuatro competencias básicas: abstracción, pensamiento sistémico, experimentación y colaboración. En Administración autores como Meyer y Jones (1993), Serva y Fuller (2004) y Benek-Rivera y Mathews (2004), entre otros, favorecen la incorporación del aprendizaje activo. El aprendizaje activo es definido como "cualquier actividad que substancialmente involucra a los estudiantes con los contenidos del curso através de hablar y escuchar, escribir, leer, y reflexionar" (Meyers \& Jones, 1993, 13).

Algunas de las estrategias dentro del aprendizaje activo que incorporan activamente a los estudiantes en su proceso de aprendizaje son: la enseñanza centrada en el estudiante (Weimer, 2002); el aprendizaje transformativo (Cranton, 1994); aprendizaje con casos (Mauffette-Leenders, Erskine, \& Leenders, 2001); aprendizaje colaborativo (Bruffee, 1993) y el aprendizaje basado en problemas (Boud \& Feletti, 1997; Savin-Baden, 2000), entre otros.

En este contexto, Jonassen y Rohrer-Murphy (1999) hacen una propuesta para construir ambientes de aprendizaje constructivistas con sustento en la Teoría de la actividad.

\subsubsection{Teoría de la actividad}

Esta teoría tiene sus raíces en la filosofía alemana clásica de Kant y Hegel, la cual enfatiza el desarrollo histórico de las ideas y en el rol activo y constructivo del ser humano. Esta filosofía es la base de posiciones filosóficas más contemporáneas como las de Marx y Engels y la psicología soviética cultural-histórica de Vigostky y otros.

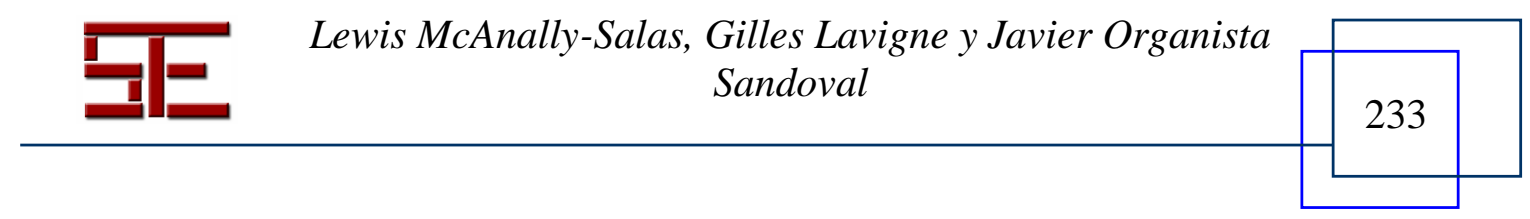




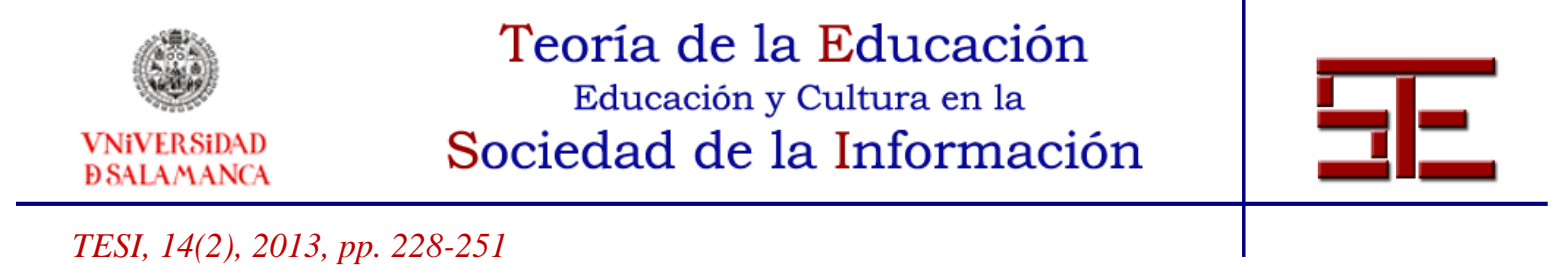

Bajo esta propuesta teórica, los autores proponen que la unidad de análisis más apropiada es la actividad, y los componentes de cada actividad se organizan en sistemas de actividades (Figura 1).

\section{Sistema de actividades}

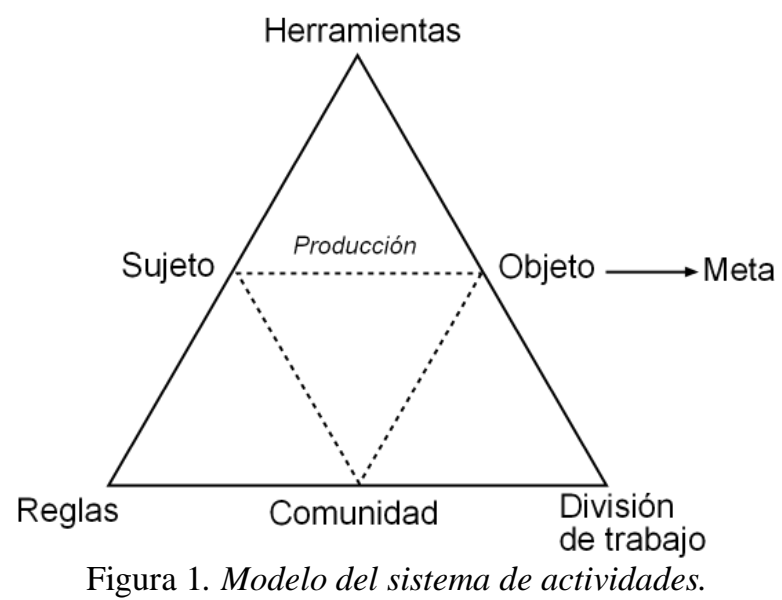

El enfoque principal en el análisis del sistema de actividades está en el triángulo superior, relacionado con la producción de algún objeto. La producción de cualquier actividad involucra un sujeto, el objeto de la actividad, las herramientas que se utilizan y las acciones y operaciones que afectan el resultado. Los sujetos de cualquier actividad son el individuo o el grupo de actores involucrados en la actividad. Las herramientas pueden ser cualquier cosa utilizada en el proceso de transformación, pueden ser físicas (destornilladores, computadoras, radios) o mentales (modelos heurísticos, de diseño educativo). El uso de herramientas determinadas por la cultura da forma a la forma como las personas piensan y actúan.

Las herramientas alteran la actividad, pero, a su vez, la actividad altera las herramientas. Por ejemplo, usar un modelo de indagación para el diseño resultará en materiales instruccionales (objetos) muy diferentes que si utilizáramos un modelo de instrucción directa. Aun así, el modelo de indagación utilizado se adaptará con cada nueva aplicación. No puede ser aplicado exactamente de la misma manera en contextos diferentes, como lo asumen muchos modelos de diseño tradicionales.

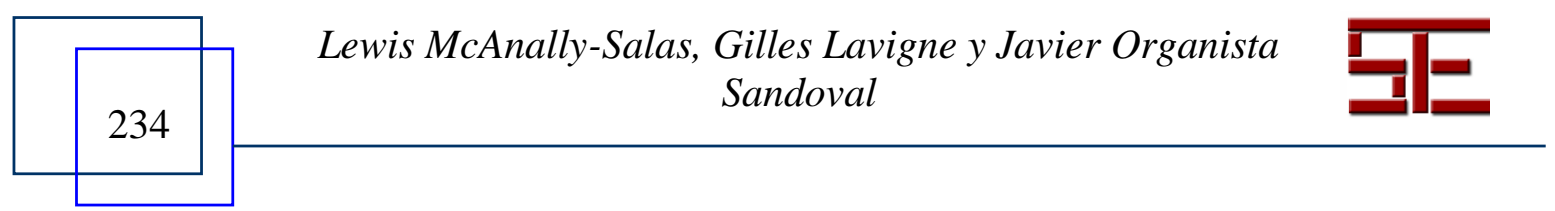




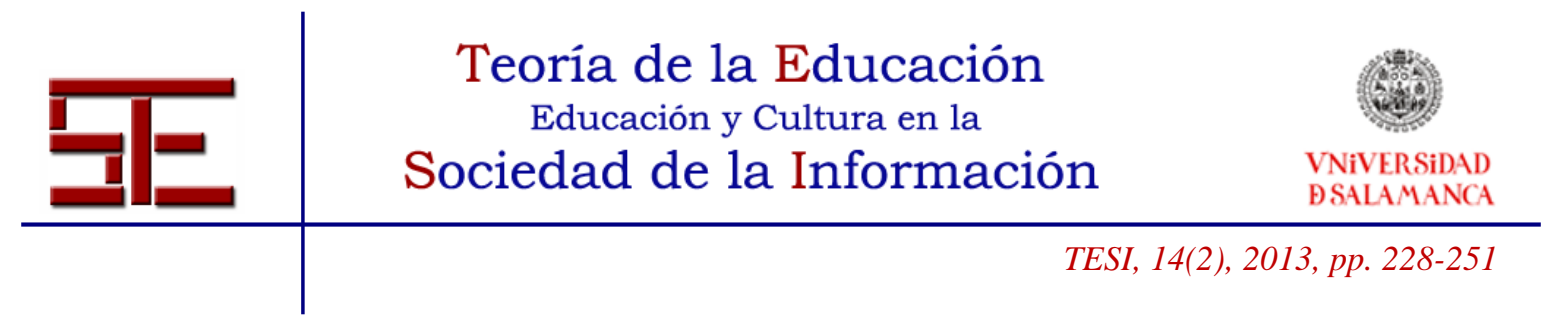

\subsubsection{Ambientes de aprendizaje constructivistas}

Bajo este contexto, en el cual los autores le dan un lugar preponderante a la actividad del sujeto dentro de un contexto, Jonassen (2006) propone un modelo para el diseño de ambientes de aprendizaje que debe considerar y enfatizar ocho características: Activo/ Manipulativo; Constructivo; Colaborativo; Conversacional; Reflexivo; Contextualizado; Complejo, e Intencional (Figura 2).

\section{Catracterísticas de ambientes de aprendizaje constructivistas}

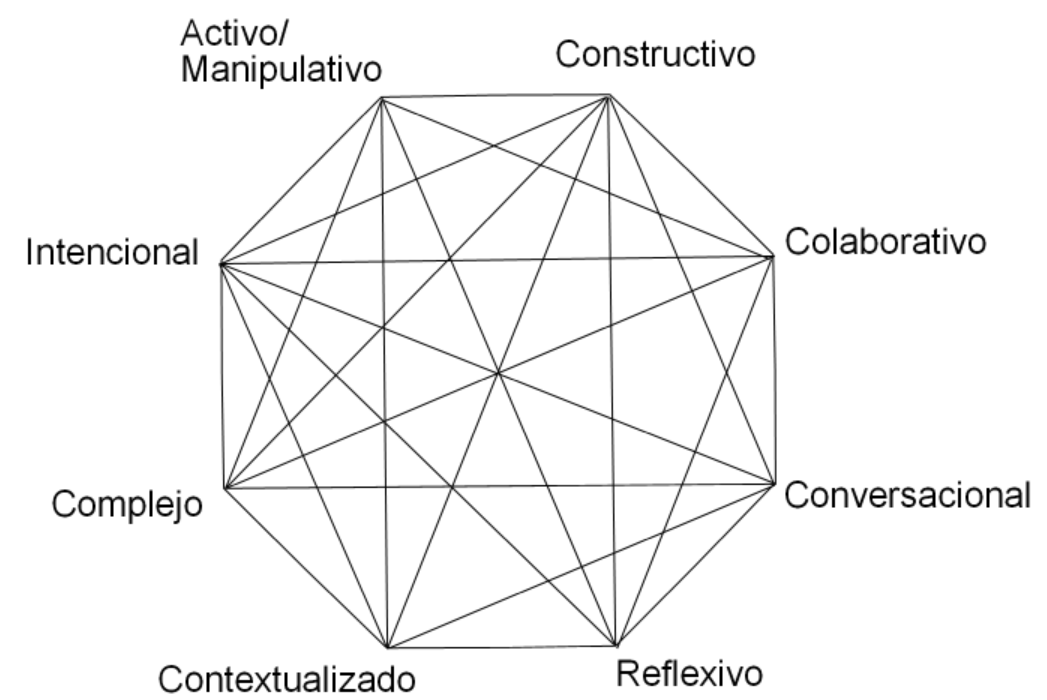

Figura 2. Características a considerar en el diseño de ambientes de aprendizaje constructivistas.

El ambiente de aprendizaje debe ser Activo/ Manipulativo para que los estudiantes se involucren en procesos de aprendizaje procesando información significativa, y donde ellos son responsables del resultado.

El ambiente de aprendizaje debe ser constructivo porque los estudiantes deben integrar nuevas ideas a sus conocimientos previos, con la intención de darles sentido, significado o reconciliar discrepancias.

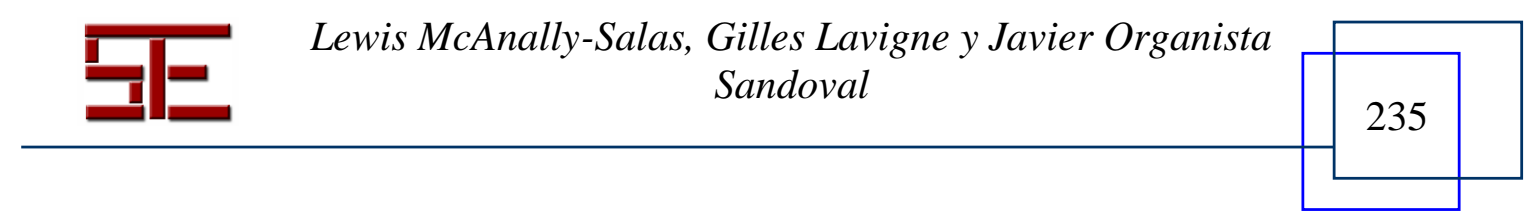




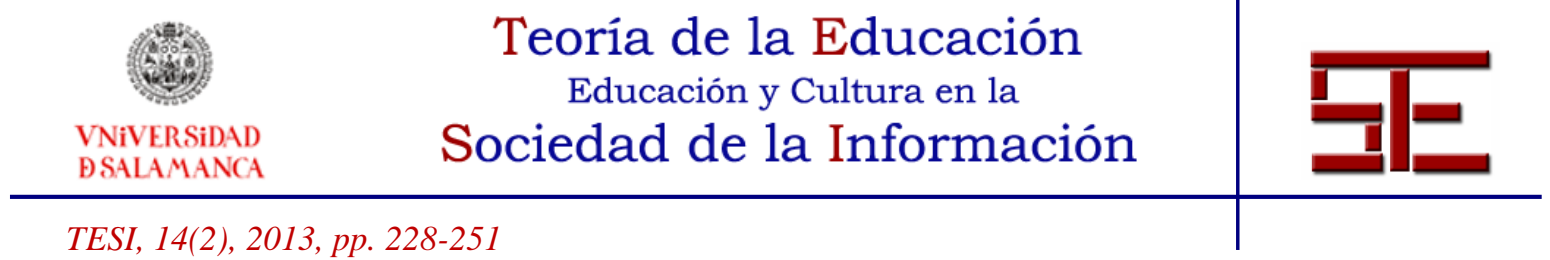

Colaborativo porque los estudiantes de manera natural tienden a construir comunidades de aprendizaje y conocimiento, aprovechando las habilidades de los demás, aportando apoyo social y modelando y observando la contribución de los demás miembros.

Debe ser intencional ya que toda la actividad humana está orientada a metas. Así, cuando los estudiantes están activamente y deseosos de lograr una meta cognitiva, piensan y aprenden mejor.

Para Jonassen (2006), Cunha y Cabral-Cardoso (2004), uno de los peores pecados intelectuales que los profesores cometen es sobre, simplificar la mayoría de las ideas para hacerlas más fácilmente transmisibles a los estudiantes. Además de descontextualizar las ideas y conceptos, son destiladas por el profesor para darlas digeridas a los estudiantes. Lo que aprenden los estudiantes es que el mundo es predecible y simple, mientras que la realidad no lo es, por eso el ambiente de aprendizaje debe reflejar la Complejidad.

La investigación ha mostrado que las tareas de aprendizaje que estén Contextualizadas en ambientes reales donde su aplicación sea significativa son comprendidas mejor y son más fácilmente transferidas a contextos distintos (Cunha et al., 2004; Serva \& Fuller, 2004). El que en el ambiente de aprendizaje se promueva la Conversación es debido a que el aprendizaje es un proceso dialógico inherentemente social. La tecnología puede favorecer estos procesos conversacionales por su potencial de comunicar estudiantes en cualquier localidad geográfica, favoreciendo las diversas maneras de ver el mundo y las diversas maneras en la solución de problemas cotidianos.

A los estudiantes se les debe solicitar Reflexión sobre lo que han hecho y hacen, cómo toman decisiones, las estrategias que utilizan, las respuestas que encuentran, etc. En este proceso ellos tendrán una mejor comprensión y serán más capaces de utilizar el conocimiento que han construido en situaciones distintas (Fink, 2003; Marzano et al., 1988; McTighe \& Wiggins, 1999).

Construir este ambiente de aprendizaje, con estrategias de corte constructivista, demanda mayor tiempo de dedicación de clase a las actividades asignadas a los estudiantes. Aunque normalmente no es posible incrementar las horas asignadas a la materia, sí es posible disminuir el tiempo dedicado a la exposición de los contenidos teóricos de los cursos. Disminuir los tiempos de exposición de contenidos es posible hacerlo bajo tres

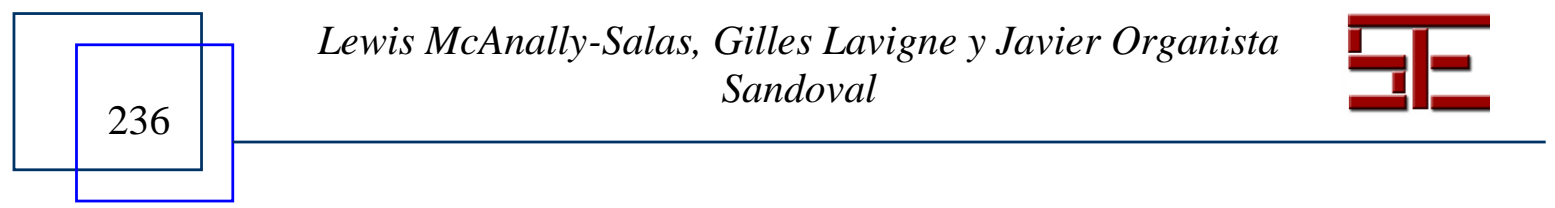




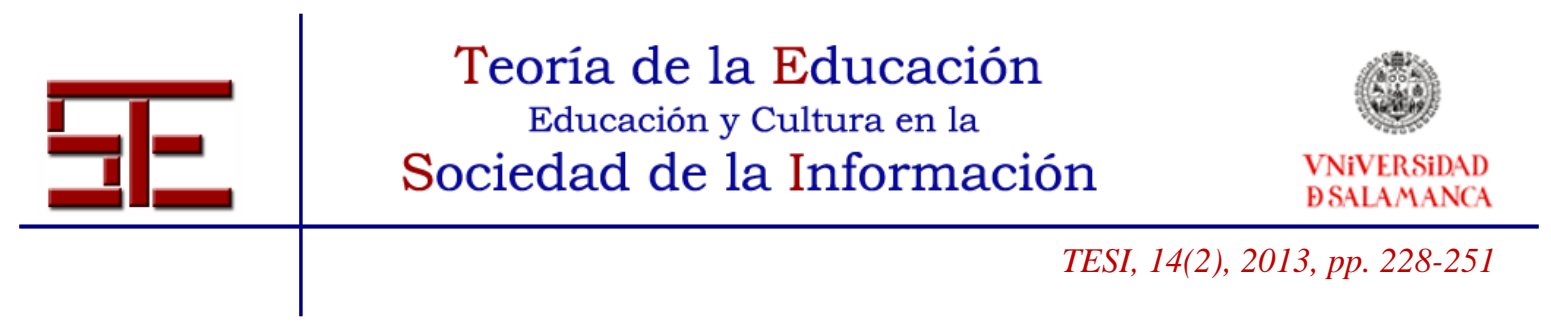

consideraciones: utilizar el modelo almenado $^{1}$ (castle top) que propone Fink, la división del conocimiento del curso en explícito y tácito, y el uso de tecnología educativa para exponer los contenidos.

\subsubsection{Modelo almenado}

Fink (2003) propone su modelo partiendo del postulado de Walvood y Anderson (Walvoord \& Anderson, 1998, 53-55) de que todos los profesores se enfrentan a dos tareas comunes; 1) que sus estudiantes aprendan el contenido de su curso y 2) que aprendan a utilizar este contenido de alguna manera. El principal problema, comentan los autores, es que los profesores pasan la mayor parte del tiempo de clase enfrascados en la primera tarea, cubriendo el contenido, dejando muy poco tiempo para enseñarles la segunda, el uso significativo del conocimiento.

El modelo almenado se utiliza como plantilla para las estrategias de enseñanza. El autor propone separar las actividades propias del aula y las actividades a realizar fuera de ella (ver Figura 3). La propuesta en este caso no es, a diferencia de Fink, hacer una diferencia de actividades dentro y fuera del aula, sino hacer una diferencia entre el conocimiento explícito y tácito que se incluyen en los contenidos y metas de aprendizaje del curso.

\begin{tabular}{|c|c|c|c|c|c|}
\hline $\begin{array}{l}\text { Actividad } \\
\text { en el aula }\end{array}$ & & $\begin{array}{l}\text { Actividad } \\
\text { en el aula }\end{array}$ & & $\begin{array}{l}\text { Actividad } \\
\text { en el aula }\end{array}$ & \\
\hline & $\begin{array}{l}\text { Actividad } \\
\text { fuera } \\
\text { del aula }\end{array}$ & & $\begin{array}{l}\text { Actividad } \\
\text { fuera } \\
\text { del aula }\end{array}$ & & $\begin{array}{l}\text { Actividad } \\
\text { fuera } \\
\text { del aula }\end{array}$ \\
\hline
\end{tabular}

Figura 3. Modelo almenado, como plantilla para estrategia de enseñanza (modificado de Fink, 2003).

\subsubsection{Tipos de conocimiento y la mediación de la tecnología.}

Independientemente de la estrategia de enseñanza utilizada, el conocimiento lo podemos clasificar en conocimiento explícito y conocimiento tácito (Nonaka \& Takeuchi, 1995). Para Lam (2000) las diferencias fundamentales entre ellos corresponden a tres aspectos: 1) la posibilidad de su codificación y los mecanismos para su transferencia, es decir, las complejidades subyacentes a su representación gráfica y distribución, 2) los métodos para

${ }^{1}$ Las almenas son prismas que coronan los muros de las antiguas fortalezas para resguardarse en ellas los defensores

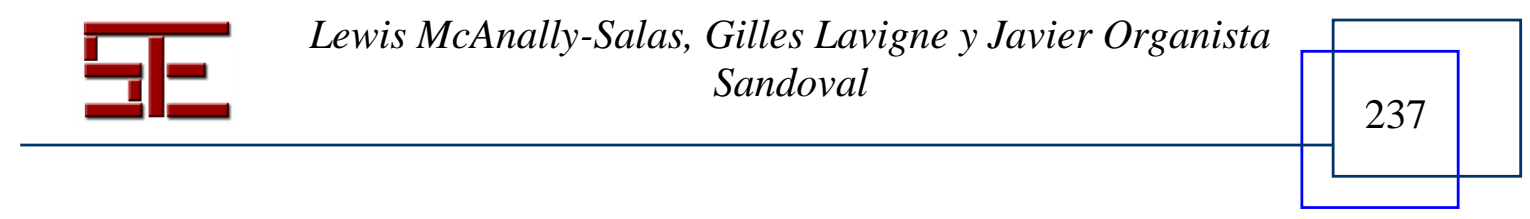




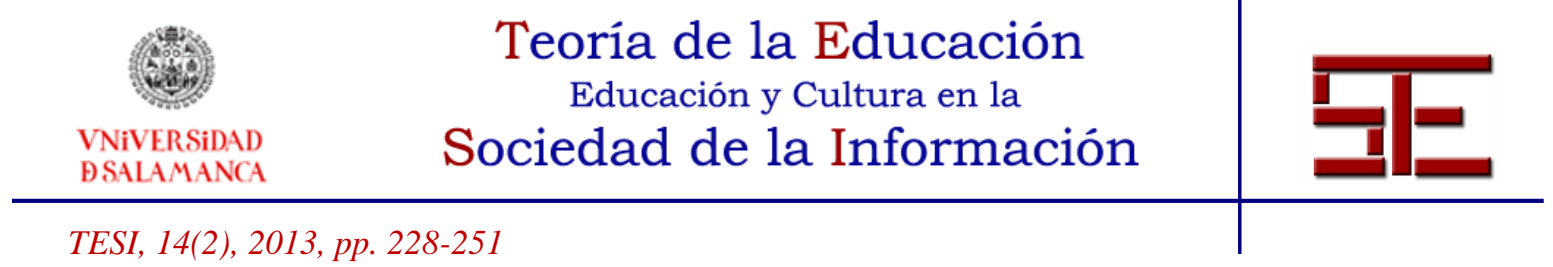

adquirir y acumular este conocimiento y 3) potencial de agregación y modos de apropiación.

En relación al primer aspecto, mientras que el conocimiento explícito es de fácil codificación y transferencia, el conocimiento tácito es intuitivo y desarticulado, está orientado a la acción y tiene cualidades personales que dificultan su codificación, por lo que es difícil su formalización y comunicación. Este conocimiento está representado por modelos mentales tales como esquemas, paradigmas, perspectivas, pensamientos y puntos de vista ayudan al individuo a percibir y a definir el mundo. Por otro lado, el elemento técnico del conocimiento tácito incluye un saber cómo concreto, habilidades relativas a cierto arte y oficio. La clave para la innovación es la movilización de los esquemas mentales en procesos que generan nuevos conocimientos. El conocimiento tácito es creado aquí y ahora en un contexto específico y pragmático. Compartir el conocimiento tácito entre individuos requiere una clase de procesamiento simultáneo de las complejidades de temas compartidos por los individuos (Nonaka et al., 1995).

Segundo, el conocimiento explícito puede ser generado a través de la deducción lógica y adquirido por el estudio formal, mientras que el conocimiento tácito solo puede ser adquirido por la experiencia personal en un contexto relevante. La transferencia del conocimiento tácito suele requerir más tiempo que el explícito, ya que es necesario tener tiempo para la manipulación, aplicación y recuperación del conocimiento que se ubica más allá del contenido mismo.

Tercero, el conocimiento explícito puede ser estar agregado en una ubicación específica, almacenado en formas objetivas y ser apropiado sin la participación del sujeto conocedor. El conocimiento tácito, en cambio, es contextual y personal, está distribuido y no puede ser agregado fácilmente, aprovecharlo en su totalidad requiere el involucramiento y cooperación del sujeto conocedor. Hasta ahora la tecnología se ha constituido en un excelente medio para comunicar, transferir y recuperar el conocimiento codificado y almacenado, sin embargo, el reto consiste en crear mecanismos y estrategias de enseñanza virtual que puedan ser retransmitidas en tiempo real, a través de las cuales, los individuos puedan enriquecer su conocimiento tácito.

Como se puede observar en la Figura 4, en el modelo almenado, la transmisión del conocimiento tácito corresponde al tiempo en el aula o laboratorio, mientras que el conocimiento explícito corresponde a las actividades fuera del aula, en nuestro caso en la

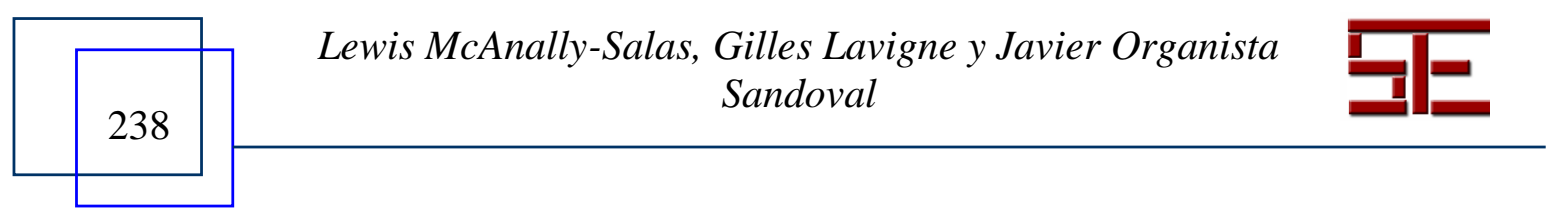




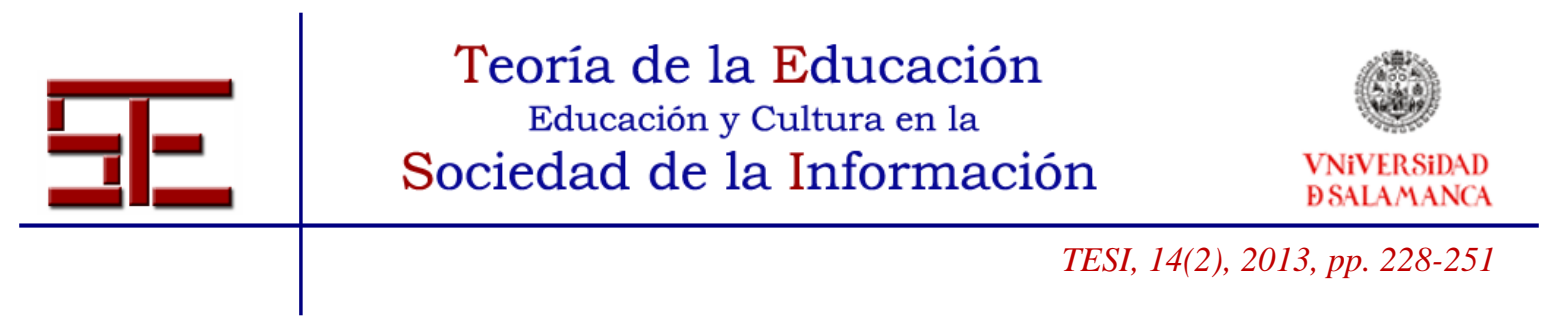

adquisición de conocimiento vía tecnologías educativas, con las consideraciones que se plantean a continuación.

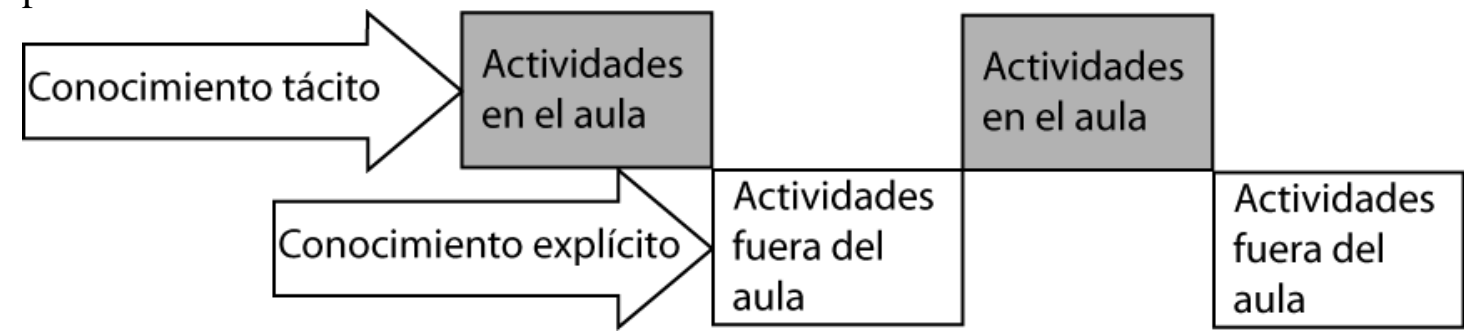

Figura 4. Modelo almenado, señalando las actividades que corresponden a conocimiento tácito y

explícito (modificado de Fink, 2003).

\subsubsection{Tecnología educativa}

De la amplia variedad de tecnologías disponibles para fines educativos, las computadoras se reconocen, desde hace muchos años, como excelentes herramientas para la transmisión de contenidos e instrucción, pueden repetir los temas, indicaciones, presentaciones, explicaciones, etc., cuantas veces sea necesario. La posibilidad de incorporar color, audio, video, animaciones y multimedia en general permite enriquecer la interacción con los contenidos, mejorar la comprensión, incentivar la motivación, ampliar la percepción y aceptación de quien aprende. Con base en estas consideraciones, la computadora se convierte en una de las herramientas más importante para la adquisición de conocimiento declarativo o explícito.

En este trabajo se asume que la incorporación de tecnologías educativas para la exposición de contenidos explícitos permite al estudiante adquirir este contenido fuera del aula, a su propio horario y conveniencia, dejando el tiempo en el aula para la interacción cara a cara, personalizada y grupal, esencial para adquirir el conocimiento tácito. Por otra parte, se pueden diseñar modelos simulados a través de los medios tecnológicos que permitan a los estudiantes situarse en un medio ambiente virtual que les permita interactuar, manipular, intercambiar y modelar como prácticas esenciales para desarrollar el conocimiento y experiencia que producen el conocimiento tácito (Schön, 1987).

Además de las ventajas señaladas, la utilización de medios tecnológicos para presentar y distribuir el contenido involucra un factor de compresión, es decir, el tiempo requerido para la presentación de contenidos se reduce con el uso adecuado de la tecnología. Esta

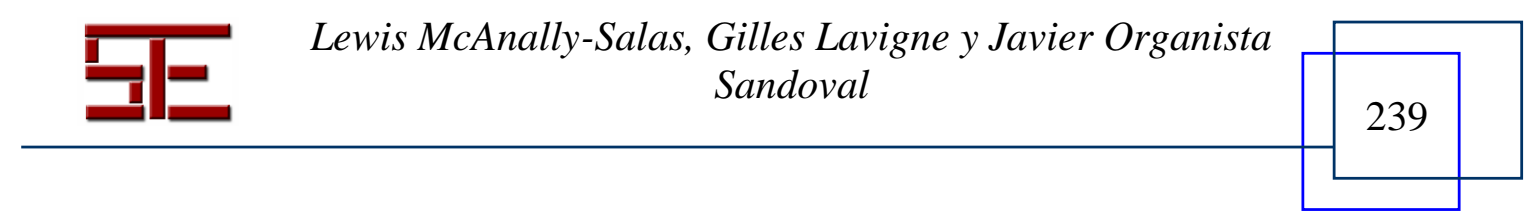




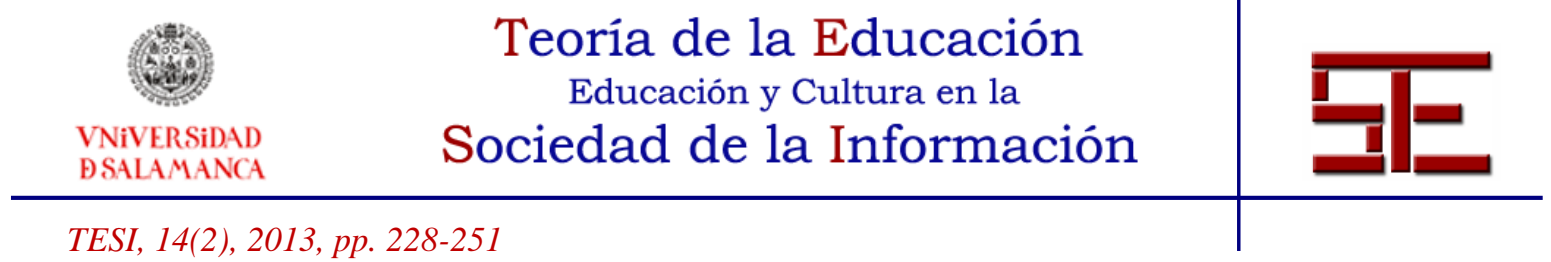

reducción es explicada por Belanger y Jordan (2000) por dos factores: 1) Debido a que, durante el diseño educativo, la selección de los mismos disminuye la paja de los contenidos y elimina los procesos normales de socialización antes y después de sesiones cara a cara y 2) Un ritmo más acelerado de aprendizaje, por las alternativas multisensoriales de los contenidos bajo una aproximación más holista del proceso de aprendizaje. La utilización de factores de compresión permite calcular las horas comprimidas, es decir, el tiempo real en que puede ser presentado un contenido utilizando un medio tecnológico.

Con la estimación del porcentaje de tiempo de exposición $\left(H_{E C}\right)$, es posible calcular las horas comprimidas $(H C)$ según la propuesta de Belanger y Jordan (2000). En el cálculo de las horas comprimidas se utiliza la fórmula

$H_{E C}-\left[H_{E C} \times F C\right]=H C$

donde $H_{E C}$ representa las horas de exposición de contenidos, $F C$ es el factor de compresión y $H C$ son las horas comprimidas.

Los factores de compresión dependen de la tecnología utilizada, Belanger y Jordan (2000) consideran: Entrenamiento Basado en Computadora (IBC); Instrucción Asistida por Computadora (IAC); Entrenamiento Basado en Web (EBW); Tele Conferencias (TC), Tele Entrenamiento por Video (TEV); y Videocintas [Tabla 1].

Tabla 1. Tasa de compresión de diferentes medios para la distribución de contenido (modificada de

Belanger \& Jordan, 2000)

\begin{tabular}{ll} 
Medio de distribución & Factor de compresión \\
\hline EBC & $35 \%$ \\
IAC Inteligente & $35 \%$ \\
EBW & $35 \%$ \\
$\boldsymbol{T C}$ & $25 \%$ \\
$\boldsymbol{T E} \boldsymbol{V}$ & $20 \%$ \\
Videocintas & $20 \%$ \\
\hline
\end{tabular}

$240 \quad$ Lewis McAnally-Salas, Gilles Lavigne y Javier Organista




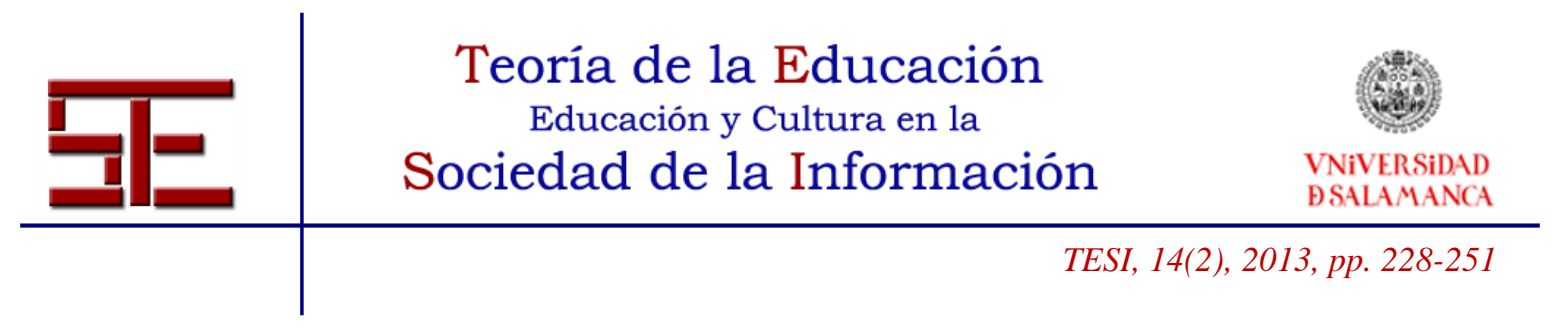

Evidentemente, entre mayor sea el tiempo de exposición de contenidos, mayor el beneficio de utilizar tecnología para su aprendizaje. En un estudio realizado en tres universidades mexicanas (McAnally-Salas, Navarro, \& Rodríguez-Lares, 2005), se encontró que los profesores dedican alrededor del $60 \%$ de su tiempo exponiendo contenidos. Lo anterior significa que un curso de 10 horas de contenido expositivo o explícito, el contenido puede ser cubierto en tan solo 4:48 horas utilizando tecnología con un factor de compresión del 20\%, como el TEV o las Videocintas, o en 3:54 horas utilizando tecnologías con un factor de compresión del 35\% como EBC, IAC y EBW. Esto equivale a disminuir el tiempo programado para contenidos en 5:12 y 6:06 horas respectivamente, tiempo liberado que puede ser utilizado en actividades de transferencia de conocimiento tácito, asociado fuertemente a la interacción, característica de estrategias de aprendizaje activo.

\section{2.- EL CASO DE LOS CURSOS DE CULTURA ORGANIZACIONAL}

La popularidad del concepto de cultura organizacional y simbolismo organizacional se ha incrementado desde la década de los 80. La relación entre cultura y análisis organizacional es tratada con profundidad por Smircich (1983), esta autora identifica, y tipifica, cinco temas de investigación relacionados con la administración y la organización que resultan de la intersección de los conceptos de cultura y organización. Estos cinco grandes temas toman el concepto de cultura de la antropología, y el concepto de organización desde la teoría organizacional. La complejidad de cada uno de estos conceptos hace evidente que se pueden tener diversas aproximaciones, por lo que estas cinco visiones investigativas son distintas, dependiendo de cómo se visualizan y abordan estos conceptos.

\subsubsection{La propuesta de Lundberg}

Craig Lundberg (1996) considera que para diseñar cursos de cultura organizacional es necesario hacerlo bajo seis consideraciones básicas sobre las que el profesor, en el proceso del diseño, va tomando algunas decisiones entre las alternativas que se le presentan. Estas seis dimensiones y sus alternativas están relacionadas con; la naturaleza del fenómeno, la posición paradigmática, las alternativas ideológicas, los modelos de aprendizaje, el dinamismo cultural y el nivel de intervención (Tabla 2).

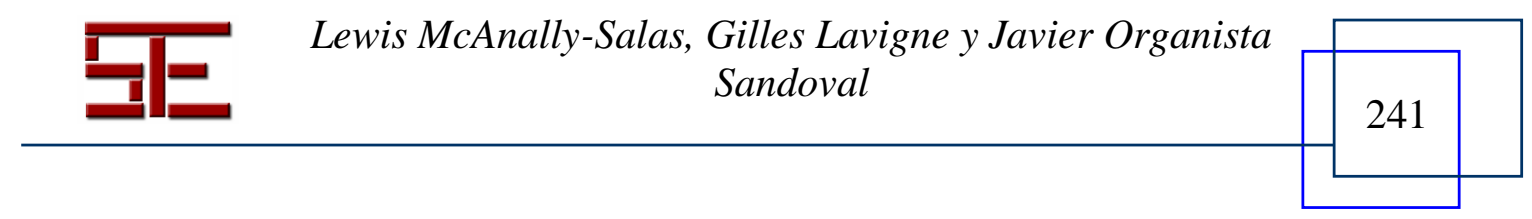




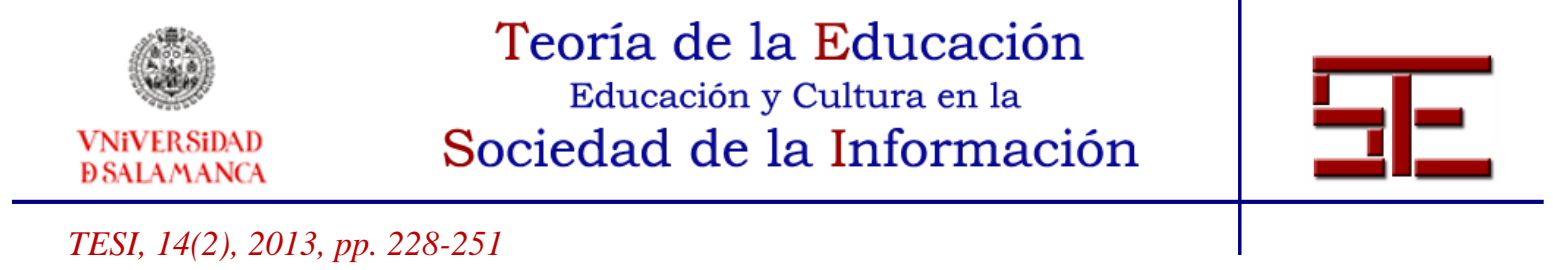

Tabla 2. Dimensiones básica para el diseño de cursos de cultura organizacional (modificada de Lundberg, 1996)

\begin{tabular}{|c|c|c|c|c|c|}
\hline $\begin{array}{l}\text { Naturaleza } \\
\text { del fenómeno }\end{array}$ & $\begin{array}{c}\text { Alternativas } \\
\text { paradigmáticas }\end{array}$ & $\begin{array}{l}\text { Alternativas } \\
\text { ideológicas }\end{array}$ & $\begin{array}{l}\text { Modelos de } \\
\text { aprendizaje }\end{array}$ & Dinamismo & $\begin{array}{c}\text { Nivel de } \\
\text { intervención }\end{array}$ \\
\hline $\begin{array}{c}\text { Real } \\
\text { (objetivo) }\end{array}$ & Integración & $\begin{array}{c}\text { Administrativa } \\
\text { o Gerencial }\end{array}$ & $\begin{array}{l}\text { Conocimiento- } \\
\text { Aplicación }\end{array}$ & $\begin{array}{l}\text { Estático o } \\
\text { transversal }\end{array}$ & Caliente \\
\hline $\begin{array}{c}\text { Creado } \\
\text { (subjetivo) }\end{array}$ & $\begin{array}{c}\text { Diferenciación } \\
\text { Ambigüedad }\end{array}$ & $\begin{array}{c}\text { Cientificista } \\
\text { Humanística } \\
\text { Crítica }\end{array}$ & $\begin{array}{l}\text { Experiencia- } \\
\text { Conocimiento }\end{array}$ & $\begin{array}{l}\text { Dinámico o } \\
\text { longitudinal }\end{array}$ & $\begin{array}{l}\text { Tibio } \\
\text { Frío }\end{array}$ \\
\hline
\end{tabular}

Lundberg divide las seis dimensiones básicas en dos grupos de tres, las primeras tres las llama consideraciones filosóficas, y las tres últimas consideraciones pedagógicas.

La naturaleza del fenómeno se relaciona con las posiciones para entender la cultura organizacional. Si se entiende la cultura organizacional como algo dado, real, entonces lo consideramos como objetivo. Por otra parte, si la cultura organizacional se entiende como algo que se construye, que es creado, entonces se considera como algo subjetivo. La posición paradigmática considera los tres paradigmas principales que dominan los estudios de cultura organizacional. Paradigma de la integración, que considera la predominancia de la cultura en términos de consistencia, claridad y amplio consenso en la organización. Es decir, los miembros de la organización coinciden, más o menos, en su cultura y existe poca ambigüedad. En contraste, el paradigma de la diferenciación, que considera la cultura organizacional como una serie de subculturas, cada una con sus propios consensos en significados y que pueden estar en armonía, conflicto o indiferentes ante ellas. El paradigma de fragmentación considera la ambigüedad como inevitable y dominante en la vida de la organización. Los consensos y los disensos coexisten en constante fluctuación por cambios circunstanciales en los eventos de la organización.

Las alternativas ideológicas, entendidas como el conjunto de ideas que están al servicio de un grupo de interés que las adopta y promueve como parte de su visión del mundo y la realidad. Consideramos cuatro grandes ideologías (Stablein, 1988). La cientificista que sigue el modelo científico de las ciencias naturales. Su interés es desarrollar estudios de cultura descriptivos, en oposición a estudios normativos. La humanista, con la idea de promover el desarrollo total del individuo. Con una visión de la cultura como vehiculo para mejorar la madurez emocional, comunicación, comportamiento ético, etc. La administrativa o gerencial es el desarrollo y apropiación del conocimiento cultural para ponerlo al servicio de la élite capitalista y administrativa. Su énfasis está en la

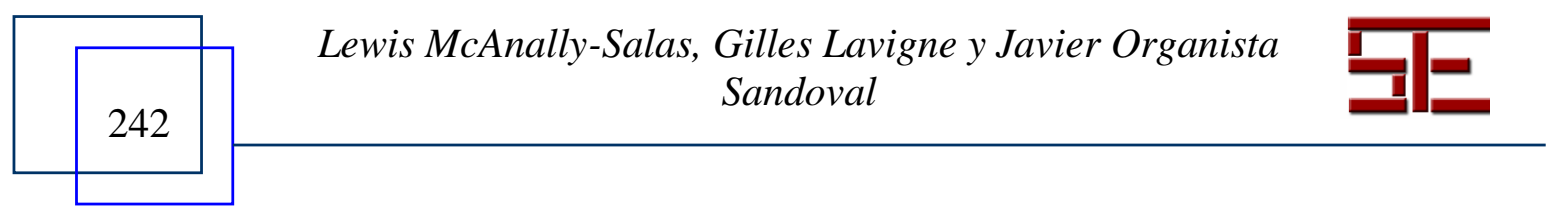




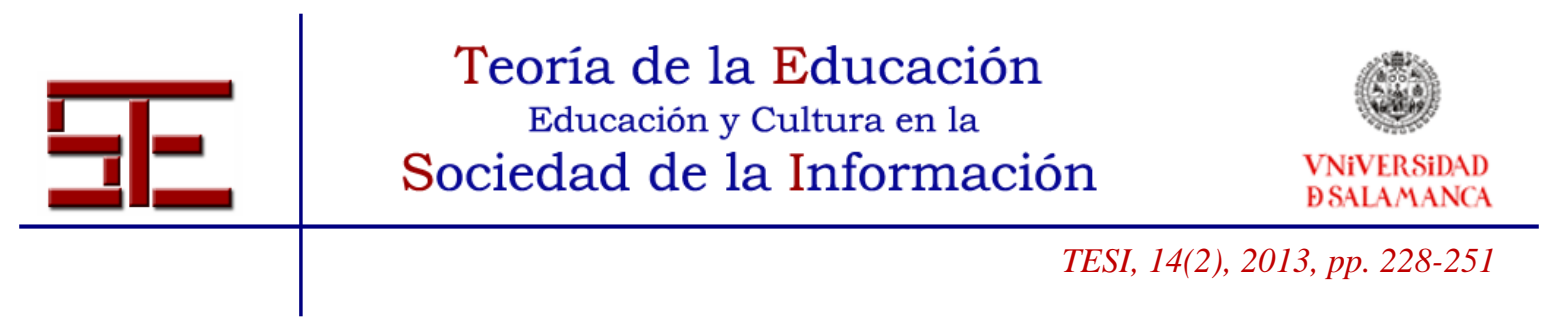

intervención directa en la organización para mejorar la productividad y las ganancias mediante el control. La crítica, que explícitamente confronta el statu quo. Se enfoca en el uso y manipulación de la cultura por los poderosos como forma de explotación, opresión y dominación.

Los modelos de aprendizaje, entendidos como la forma en que planea el docente la secuencia de las actividades. Teoría luego experiencia, en donde inicia con el conocimiento explícito y luego conocimiento tácito, o bien Experiencia luego teoría.

El dinamismo cultural se refiere a la consideración de la variable tiempo en el estudio de cultura organizacional. Si el estudio de la cultura sólo considera un punto en el tiempo, se considera un estudio estático o transversal, sin embargo, si el estudio se refiere a los cambios culturales a lo largo del tiempo, entonces estamos hablando de estudios dinámicos o longitudinales.

El nivel de intervención se refiere específicamente al tipo de experiencia o contenido que se programa en el curso, considerando el dónde y el cuándo. Así tenemos alternativas de intervención calientes porque tratan nuestra propia cultura en nuestro tiempo, o bien frías al tratar otras culturas ajenas en otro tiempo.

\subsubsection{Recomendaciones para el diseño educativo del curso}

Las seis dimensiones propuestas por Lundberg (1996) realmente son una síntesis de las diferentes posiciones que se pueden tomar. Estas seis dimensiones en principio deberían ser, en su totalidad, la columna vertebral sobre la que se articulan los contenidos, y no deben ser vistas como las alternativas para seleccionar una trayectoria única, es el abanico de la temática a tratar.

Todo indica que la propuesta de Lundberg (1996) tiene una aproximación básicamente conductista del proceso enseñanza-aprendizaje. Como se comentó anteriormente, se asume que el conocimiento está dado y a los estudiantes únicamente se les debe indicar qué es lo que deben aprender. La posición del autor es que el profesor sabe qué deben aprender los estudiantes, y cuál es el cúmulo de conocimientos que los estudiantes deben adquirir. El profesor es la fuente del conocimiento, el cual marca la pauta a seguir.

Seguir a pie juntillas los lineamientos de Lundberg (1996) marcaría una ruta al estudiante que no le permitiría, o lo haría de manera limitada, explorar otras posiciones distintas.

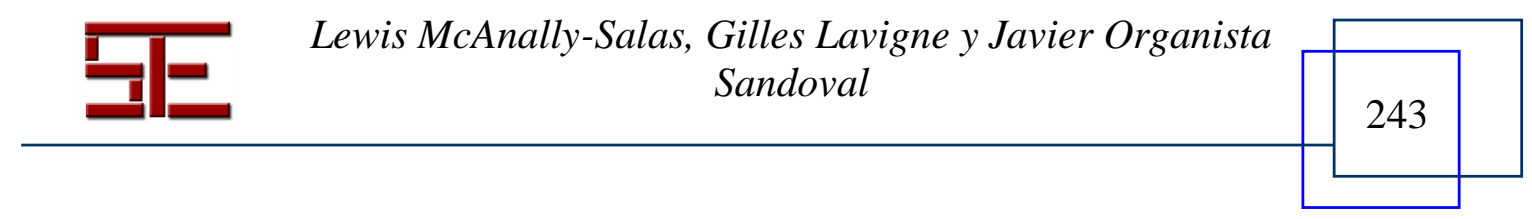




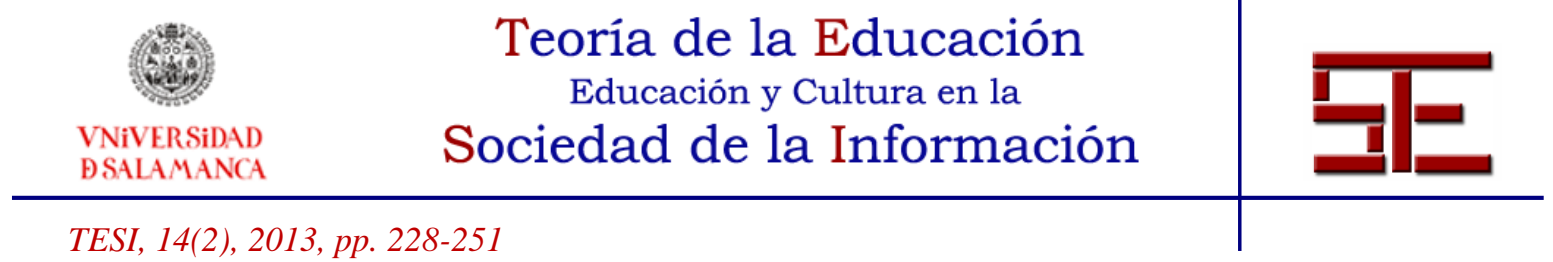

Así, si desde el inicio el profesor optó por aproximarse a la cultura organizacional como una variable que se puede controlar, ese estudiante difícilmente podrá conocer las posiciones de los autores que ven a la cultura como una metáfora raíz. Para cada una de las seis dimensiones consideradas, la prescripción de seleccionar una reduce la visión de la cultura organizacional a la visión del profesor, sin dejar oportunidad para que los estudiantes discutan, dialoguen, construyan sus propias visiones al amparo de la diversidad de autores. Lo que se plantea en este trabajo es que al asumir una aproximación constructivista del aprendizaje, es fundamental que el estudiante sea conciente de la multitud de perspectivas. De esta forma, la complejidad, como parte del ambiente normal de la organización y del trabajo profesional, es una dimensión transversal del diseño educativo del curso. Por sus propias posiciones paradigmáticas e ideológicas, resulta evidente que cada profesor tiene una posición ante la cultura organizacional, difícilmente existe la neutralidad, sin embargo, la imposición de las visiones propias no siempre es en ventaja del estudiante. Esta perspectiva propia que el profesor le va confiriendo a su curso se ve enriquecida al permitir que los estudiantes complementen/construyan sus propias visiones con perspectivas alternativas.

También es comprensible que por la cantidad de temas involucrados no es posible que el profesor cubra todas las aproximaciones y todo el contenido en un periodo escolar.

La propuesta es considerar todas las alternativas que se encuentran en el grupo de consideraciones filosóficas como ejes centrales y seguir las recomendaciones de Lundberg (1996) para las dimensiones del grupo de consideraciones pedagógicas para situaciones más puntuales, que normalmente dependen de las metas de aprendizaje y el contexto del curso.

\section{3.- CONCLUSIONES}

Transitar del paradigma centrado en la enseñanza al paradigma centrado en el aprendizaje no es cosa sencilla por la multidimensionalidad que conlleva. El diseño de cursos buscando la coherencia con una aproximación constructivista, que utilice otras teorías del aprendizaje cuando sea pertinente, permite una visión más holista e integradora que la búsqueda de diseños educativos químicamente puros.

En los últimos años se han desarrollado múltiples estrategias que se basan en principios cognoscitivistas y constructivistas que permiten ser adaptadas a una gran diversidad de condiciones y necesidades de aprendizaje. Una propuesta como la de Lundberg de finales del Siglo XX que favorece la incorporación de estrategias de aprendizaje activo en el aprendizaje de la Administración les permite a los estudiantes adquirir habilidades de

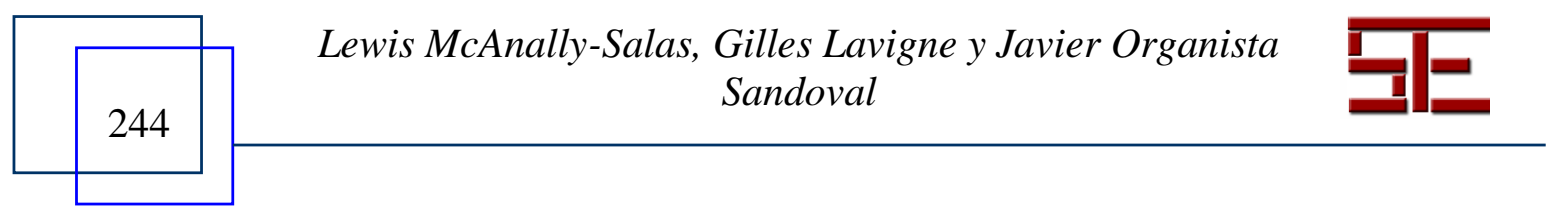




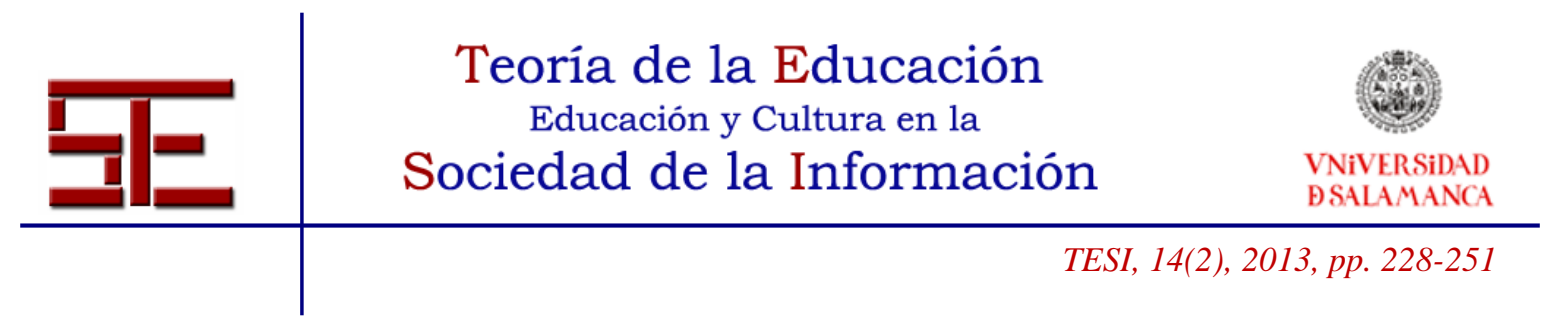

analistas simbólicos, perfectamente apropiadas para el desarrollo de profesionistas para el siglo XXI.

La definición de ambientes de aprendizaje constructivistas les permite a los profesores tener una visión global de los requerimientos para su curso si se quieren lograr aprendizajes significativos. El uso de la tecnología educativa se ha vuelto un elemento fundamental que en muchos casos le da viabilidad a las estrategias de aprendizaje. Por su importancia, la tecnología educativa tiene que ser eficientemente utilizada, identificando las circunstancias y contenidos que se benefician de su uso.

La distinción entre conocimiento explícito y tácito permite determinar las mejores condiciones y herramientas para su transmisión y adquisición, permitiendo un uso más eficiente del tiempo, espacios y recursos tecnológicos. Lo anterior se hace evidente con la incorporación del modelo almenado propuesto como plantilla para la programación de actividades y contenidos.

Para Castells $(2000,2008)$ las transformaciones fundamentales ocurridas en la sociedad y organizaciones a finales del siglo pasado ha complejizado la comprensión del mundo social y laboral. En este contexto, la cultura como reflejo de estas transformaciones juega un papel primordial para la comprensión de los retos presentes en una sociedad donde las organizaciones cuentan con modelos económicos y de gestión con elementos desconocidos hace pocas décadas. Es por lo anterior que la cultura organizacional adquiere relevancia en las carreras de Administración, ya que permite a los estudiantes entrar al proceso de comprensión de eventos y situaciones fundamentales en la vida de las organizaciones. La propuesta que se presenta como complemento al modelo de Lundberg (1996) permite a los profesores articular estrategias en el diseño de cursos que consideran las aproximaciones más actuales, ya que tomar tan literal y prescriptivamente las recomendaciones del autor dejaría huecos importantes en la comprensión de la cultura organizacional. Por lo anterior, se propone recuperar su propuesta con una aproximación más constructivista, tomando las consideraciones filosóficas como parte medular del temario, y las consideraciones pedagógicas, más dependientes de las metas de aprendizaje y del contexto del curso. Esto permitiría tener una visión más realista del estado del conocimiento en lo referente a cultura en las organizaciones.

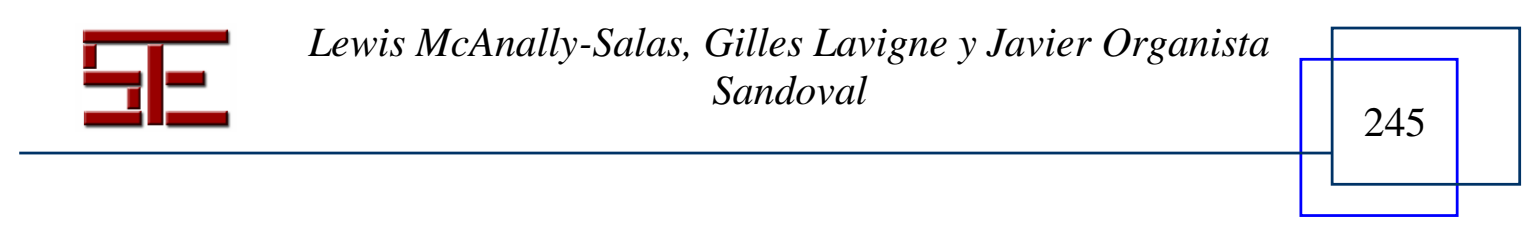




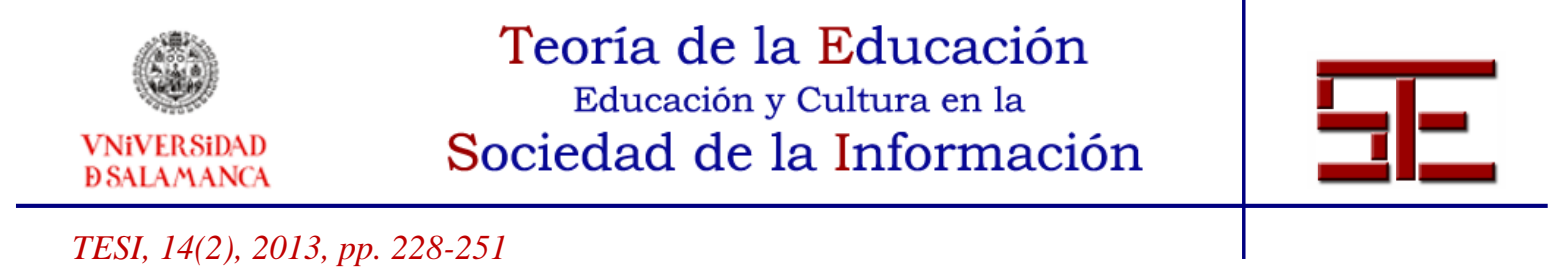

\section{4.- BIBLIOGRAFÍA}

Ally, M. (2004). Foundations of Educational Theory for Online Learning. Theory and Practice of Online Learning (pp. 3-31). Athabasca, $\mathrm{AB}_{-}$_: Athabasca University. Retrieved from_http://cde.athabascau.ca/online_book/

Belanger, F., \& Jordan, D. H. (2000). Evaluation and implementation of distance learning: technologies, tools, and techniques. Hershey, PA: Idea Group Pub.

Benek-Rivera, J., \& Mathews, V. E. (2004). Active learning with Jeopardy: Students ask the questions. Journal of Management Education, 28 (1), 104-118.

Boud, D., \& Feletti, G. (1997). The Challenge of problem-based learning (vol. 2nd). London: Kogan Page.

Bruffee, K. A. (1993). Collaborative learning: higher education, interdependence, and the authority of knowledge. Baltimore, Md: Johns Hopkins University Press.

Castells, Manuel. (2000). Globalización, Sociedad y Política En la era de la Información. Bitácora. 4, no. 1, 42-53.

- (2008) La Era de la información: economía, sociedad y cultura. vol. 1 Siglo XXI.

Clarke, T. \& Clegg, S. (1998). Changing paradigms: the transformation of management knowledge for the 21 st century. London: HarperCollins Business.

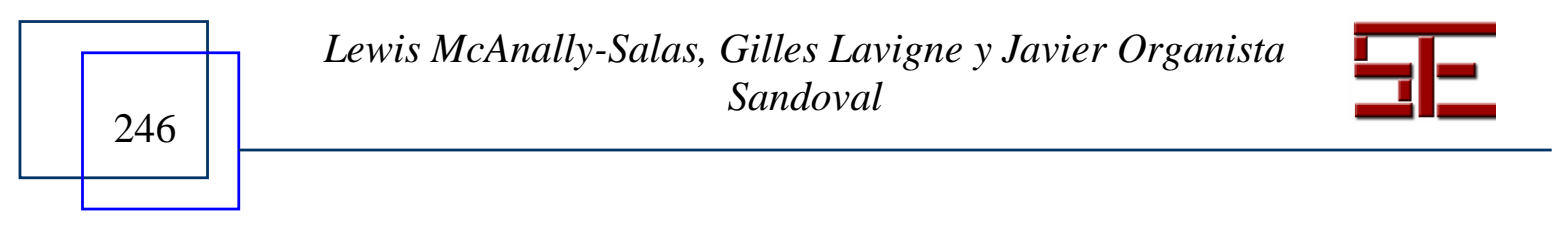




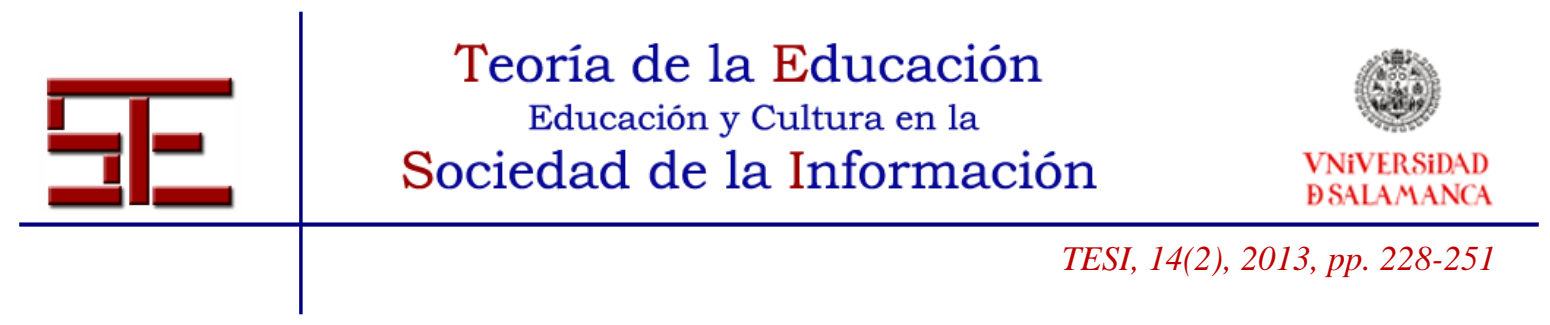

Cranton, P. (1994). Understanding and promoting transformative learning a guide for educators of adults. Jossey-Bass higher and adult education series (Vol. 1st). San Francisco: Jossey-Bass.

Cunha, M. P., Cunha, J. V. da \& Cabral-Cardoso, C. (2004). Looking for complication: Four approaches to management education. Journal of Management Education, $28(1), 88-103$.

Despres, C. \& Chauvel, D. (2000). Knowledge horizons: the present and the promise of knowledge management. Boston: Butterworth-Heinemann.

Fink, D. (2003). Creating Significant Learning Experiences: An Integrated Approach to Designing College Courses. San Francisco, CA: Jossey-Bass.

Gibbons, M. (1994). The new production of knowledge: the dynamics of science and research in contemporary societies. London: Sage.

Jonassen, D. H. (2006, May 30). Welcome to the Design of Constructivist Learning Environments (CLEs).

http://tiger.coe.missouri.edu/ jonassen/courses/CLE/main.html. Retrieved from _http://tiger.coe.missouri.edu/ jonassen/courses/CLE/_.

Jonassen, D. H. \& Rohrer-Murphy, L. (1999). Activity theory as a framework for desinging constructivist learning environments. Edcuational Technology Research and Development, 47 (1), 61-79.

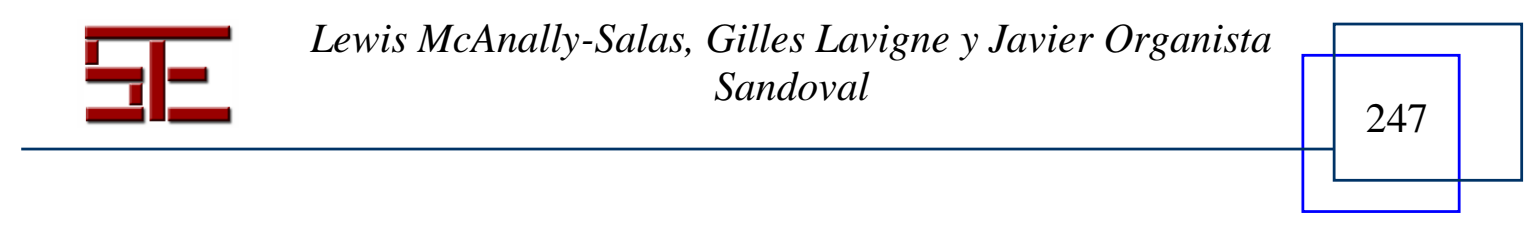




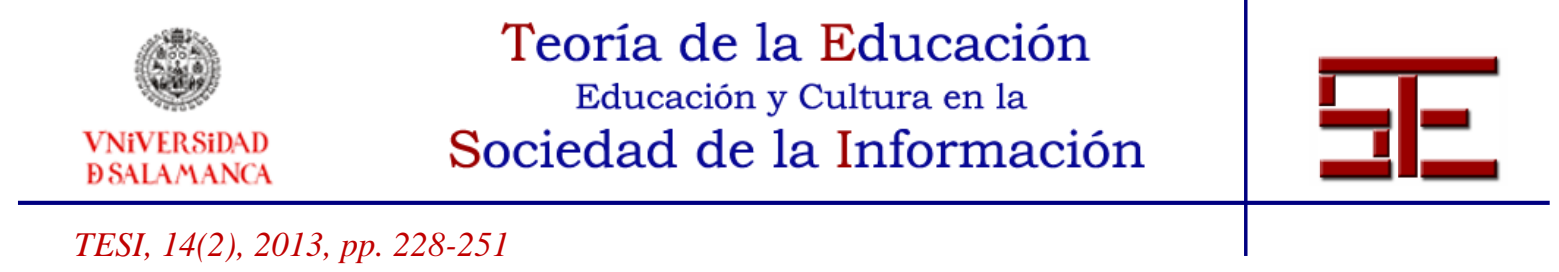

Katz, R. N. \& Associates. (1999). Tying Things Together: Advice for Practitioner. En Dancing with the devil: Information technology and the new competition in higher education. Jossey-Bass higher and adult education series (vol. 1st ed. 119-122). San Francisco, Calif: Jossey-Bass Publishers.

Katz, R. N., EDUCAUSE (Association) \& PricewaterhouseCoopers, L. L. P. (1999). Dancing with the devil: Information technology and the new competition in higher education. Jossey-Bass higher and adult education series (vol. 1st ed.). San Francisco, Calif: Jossey-Bass Publishers.

Lam, A. (2000). Tacit Knowledge, Organizational Learning and Societal Institutions: An Integrated Framework. Organization Studies, 21 (3), 487-513.

Latham, G. Latham, S. D., \& Whyte, G. (2004). Fostering integrative thinking: Adapting the executive education model to the MBA program. Journal of Management Education, 28 (1), 3-18.

Lundberg, C. C. (1996). Designing Organizational Culture Courses: Fundamental Considerations. Journal of Management Education, 20 (1), 11-22.

Marzano, R. J., Brand, R. S., Hughes, C. S., Jones, B. F., Presseisen, B. Z., Rankin, S. C. \& Suhor, C. (1988). Dimensions of thinking: A framework for curriculum and instruction. Alexandria, VA: Association for Supervision and Curriculum Development.

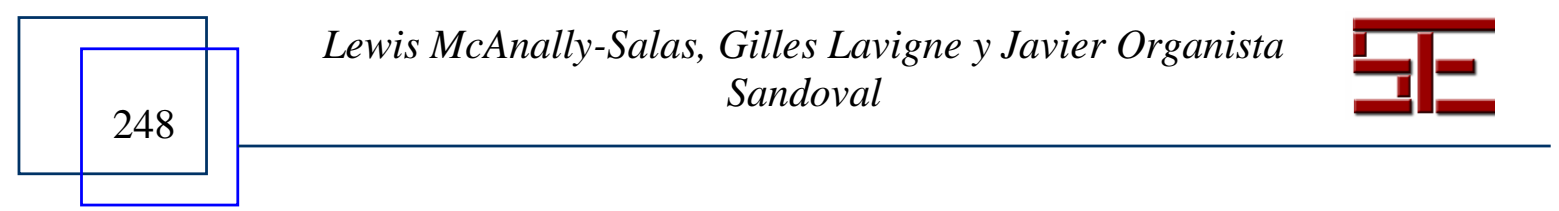




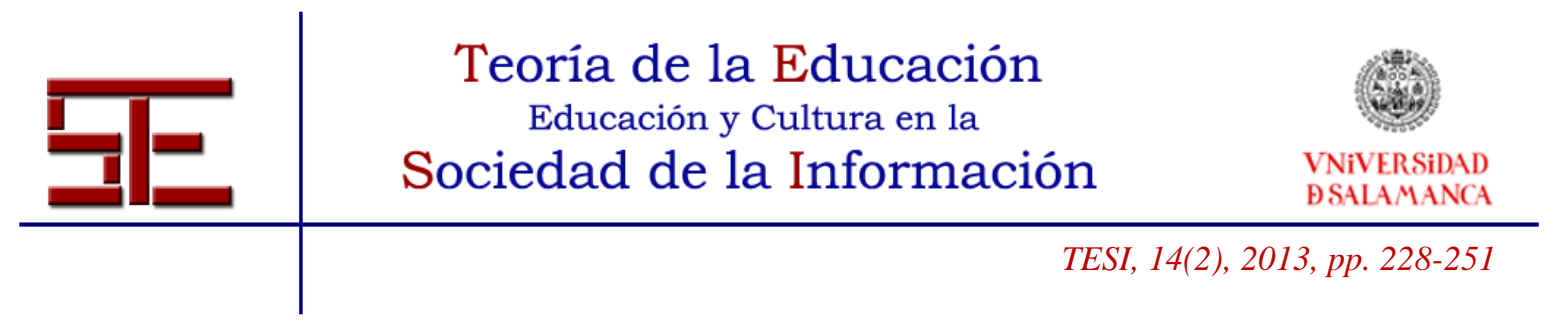

Mauffette-Leenders, L. A., Erskine, J. E. \& Leenders, M. R. (2001). Learning with cases (vol. 2nd). London, Ont: Ivey Publishing, Richard Ivey School of Business.

McAnally-Salas, L., Navarro, M. de R. \& Rodríguez-Lares, J. J. (2005). La estrategia de enseñanza y la integración de la tecnología educativa como alternativa para ampliar la capacidad de atención en la educación superior. Revista Mexicana de Investigación Educativa.

McTighe, J. \& Wiggins, G. P. (1999). Understanding by design handbook. Alexandria, Va: Association for Supervision and Curriculum Development.

Meyers, C. \& Jones, T. B. (1993). Promoting active learning: Strategies for the college classroom. San Francisco, CA: Jossey-Bass.

Nonaka, I. \& Takeuchi, H. (1995). The knowledge-creating company: how Japanese companies create the dynamics of innovation. New York: Oxford University Press.

Oblinger, D. \& Rush, S. C. (1997). Challenges of the Learning Revolution. En The learning revolution: The challenge of information technology in the academy (pp. 231-243). Bolton, Mass: Anker.

Reich, R. B. (1992a). The Education of Symbolic Amalyst (I). En The work of nations: preparing ourselves for 21st century capitalism (Vol. 1st Vintage Books, pp. 225240). New York: Vintage Books.

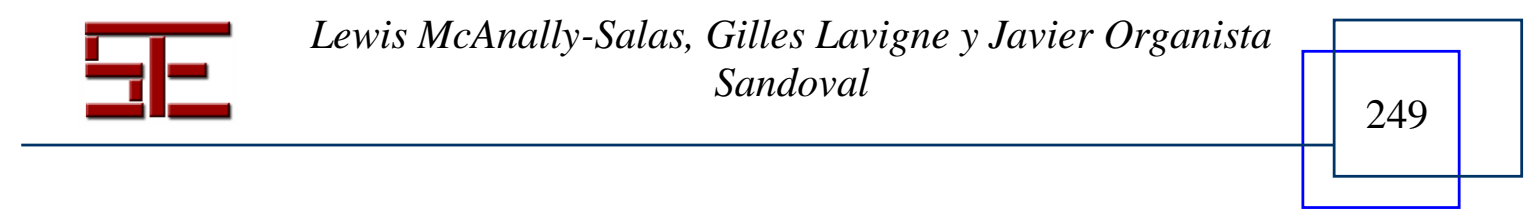




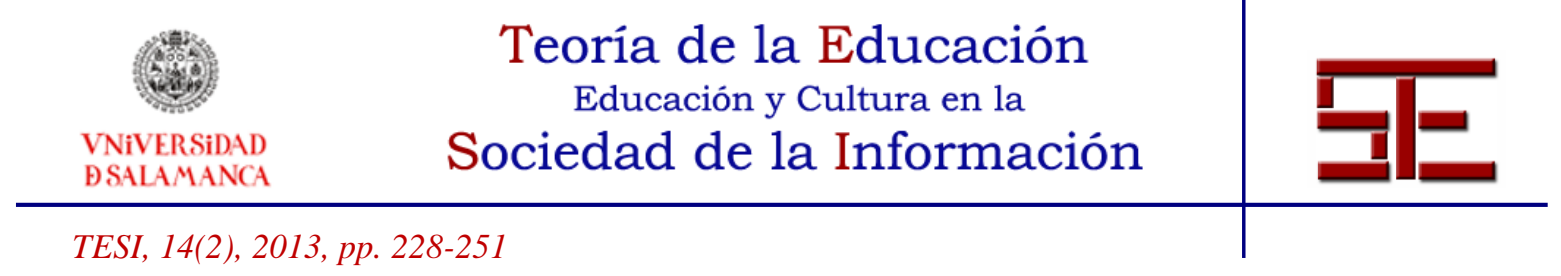

- (1992b). The Work of Nations (vol. 1st Vintage Books). New York: Vintage Books.

Roblyer, M. D. \& Edwards, J. (2000). Integrating educational technology into teaching (vol. 2nd ed). Upper Saddle River, N. J: Merrill.

Savin-Baden, M. (2000). Problem-based learning in higher education untold stories. Buckingham England: Society for Research into Higher Education \& Open University Press.

Schön, D. A. (1987). Educating the Reflective Practitioner. San Francisco, CA: JosseyBass.

Serva, M. A. \& Fuller, M. A. (2004). Aligning what we do and what we measure in Business Schools: Incorporating Active Learning and effective media use in the assessment of instruction. Journal of Management Education, 28 (1), 19-38.

Smircich, L. (1983). Concepts of Culture and Organizational Analysis. Administrative Science Quarterly, 28 (3), 339-358. doi:10.2307/2392246.

Stablein, R. E. (1988). Structure of debate in organizational studies. Perth, Australia: Annual Meeting of Australia Management Educators.

Walvoord, B. E. F. \& Anderson, V. J. (1998). Effective grading a tool for learning and assessment. The Jossey-Bass higher and adult education series (vol. 1st). San Francisco, Calif: Jossey-Bass Publishers.

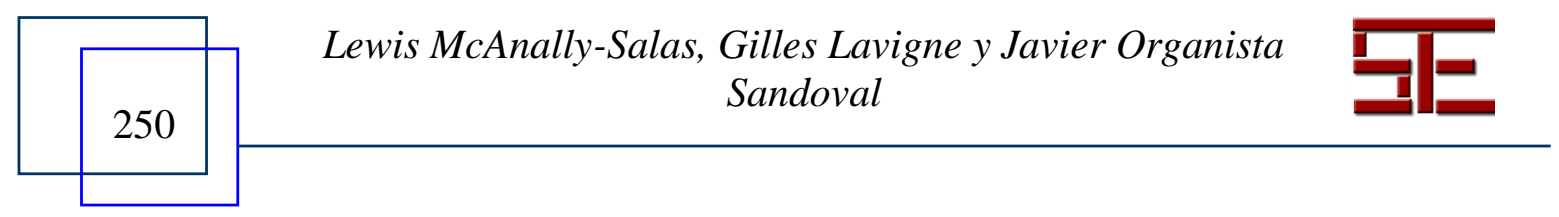




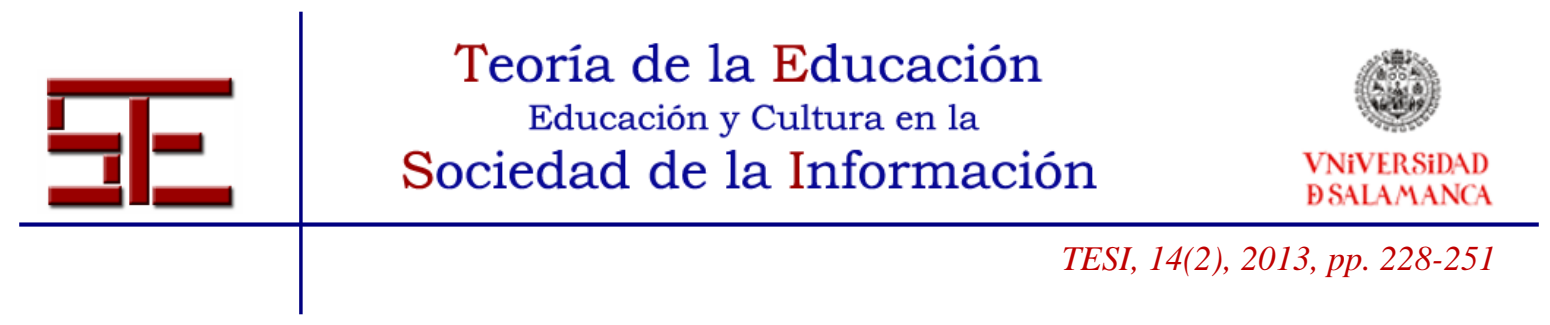

Weimer, M. (2002). Learner-centered teaching five key changes to practice. Jossey-Bass higher and adult education series (vol. 1st). San Francisco: Jossey-Bass. Retrieved from http://www.loc.gov/catdir/description/wiley035/2002005662.html; http://www.loc.gov/catdir/toc/wiley023/2002005662.html.

Para citar el presente artículo puede utilizar la siguiente referencia:

Mcanally-Salas, L., Lavign, G. y Organista Sandoval, J. (2013). Marco de referencia para diseñar cursos en administración: el caso de cultura organizacional. Revista Teoría de la Educación: Educación y Cultura en la Sociedad de la Información. 14(2), 228-251 [Fecha de consulta: $\mathrm{dd} / \mathrm{mm} / \mathrm{aaaa}]$.

http://campus.usal.es/ revistas_trabajo/index.php/revistatesi/article/view/10221/10630

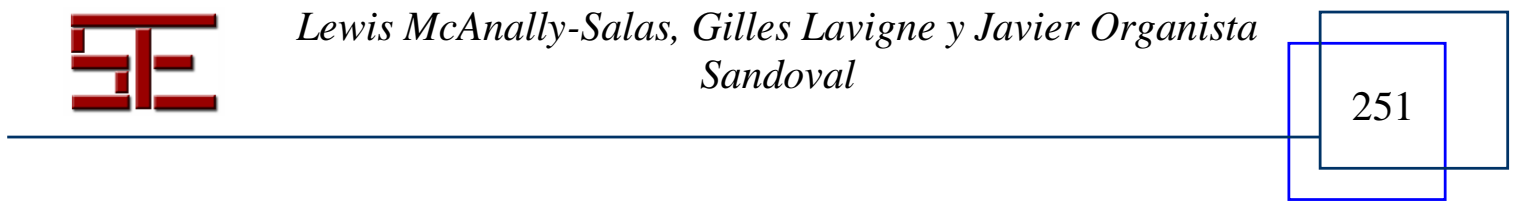

